\title{
The effective action in 4-dim CDT. The transfer matrix approach
}

\author{
J. Ambjørn, ${ }^{a, b}$ J. Gizbert-Studnicki, ${ }^{c}$ A. Görlich ${ }^{a, c}$ and J. Jurkiewicz ${ }^{c}$ \\ ${ }^{a}$ The Niels Bohr Institute, Copenhagen University, \\ Blegdamsvej 17, DK-2100 Copenhagen Ø, Denmark \\ ${ }^{b}$ IMAPP, Radboud University, \\ Niemegen, The Netherlands \\ ${ }^{c}$ Institute of Physics, Jagellonian University, \\ Reymonta 4, PL 30-059 Krakow, Poland \\ E-mail: ambjorn@nbi.dk, jakub.gizbert-studnicki@uj.edu.pl, \\ atg@th.if.uj.edu.pl, jurkiewicz@th.if.uj.edu.pl
}

ABstract: We measure the effective action in all three phases of 4-dimensional Causal Dynamical Triangulations (CDT) using the transfer matrix method. The transfer matrix is parametrized by the total 3-volume of the CDT universe at a given (discrete) time. We present a simple effective model based on the transfer matrix measured in the de Sitter phase. It allows us to reconstruct the results of full CDT in this phase. We argue that the transfer matrix method is valid not only inside the de Sitter phase ('C') but also in the other two phases. A parametrization of the measured transfer matrix/effective action in the ' $\mathrm{A}$ ' and ' $\mathrm{B}$ ' phases is proposed and the relation to phase transitions is explained. We discover a potentially new 'bifurcation' phase separating the de Sitter phase ('C') and the 'collapsed' phase ('B').

Keywords: Models of Quantum Gravity, Random Systems, Lattice Models of Gravity

ARXIV EPRINT: 1403.5940 


\section{Contents}

1 Introduction 1

2 Methodology of the transfer matrix measurements 5

3 The effective model in the de Sitter phase $\quad 6$

$\begin{array}{lll}4 & \text { Extracting the kinetic and potential terms } & 10\end{array}$

5 The transfer matrix in phase ' $A$ ' 10

6 The transfer matrix in phase ' $B$ ' 13

$\begin{array}{llr}7 & \text { Phase transitions } & 18\end{array}$

8 A new 'bifurcation' phase? 20

$\begin{array}{lll}9 & \text { Summary and conclusions } & 23\end{array}$

\section{Introduction}

The method of triangulations was introduced in the context of General Relativity by Regge [1] to discretize the continuous Hilbert-Einstein action

$$
S_{H E}[g]=\frac{1}{16 \pi G} \int d^{4} x \sqrt{-g}(R-2 \Lambda) .
$$

Continuous geometries are approximated by piecewise linear simplicial manifolds. The curvature is represented as a deficit angle concentrated at the (D-2) subsimplex. A particular realization of this idea is the method of Dynamical Triangulations (DT), where the piecewise linear simplicial manifolds are built by gluing together regular, identical simplices with identical edge lengths $a[2-6]$. The DT set of simplicial geometries is thus entirely characterized by the abstract triangulations which define how the simplices are glued together and it has been useful in Monte Carlo simulations of quantum gravity, a acting as a UV cut off. In $\mathrm{D}=2$ one could even solve the DT model analytically for gravity coupled to certain simple matter systems and the continuum limit $a \rightarrow 0$ could be obtained. These results were reproduced by conformal field theory methods (so-called 2D quantum Liouville theory) [7-10].

For higher dimensional quantum gravity the DT approach has been less successful [1121]. Firstly, there are only very few analytical results. Most investigations use Monte Carlo simulations to evaluate the path integrals. This method has also been tested and has 
proven very successful in $D=2$. Secondly, in the three- and four-dimensional DT cases the simplest versions of the lattice theory, characterized by two coupling constants, analogous to that of the continuum theory (1.1), did not show a behaviour which could be viewed as interesting from a continuum gravity point of view. Depending on the strength of the bare lattice gravitational coupling constant, the system (rotated to Euclidean time in order to allow for Monte Carlo simulations) appeared to have two phases. The weak gravity phase was dominated by the branched polymer geometries with a Hausdorff dimension $d_{H}=2$ and the strong gravity phase by collapsed geometries with possibly $d_{H}=\infty$, corresponding to universes without a linear extension. The two phases were separated by a first order phase transition [22-24].

The method of Causal Dynamical Triangulations (CDT) was introduced to cure these problems [25-28] (for pedagogical reviews see [29-31]). At this point it should be made clear that the problems encountered in DT could very well reflect the fact that there is no stand alone theory of quantum gravity based only on the metric tensor $g_{\mu \nu}$. This is in a certain way what we are trying to investigate. CDT enlarges the scope of metric theories one can reach, but eventually one might encounter some of the same problems as in DT. In CDT a notion of the proper time was introduced together with the requirement that the spatial topology of the quantum universe with respect to this proper time must be preserved in the time evolution $[32,33]$. The simplest version of the discretized theory using the CDT approach has three parameters. Apart from the two parameters present in the DT approach, related to the cosmological constant and to the gravitational constant, the additional parameter controls a possible asymmetry between the edge lengths in the spatial and time directions. In numerical simulations the topology of the manifold is assumed to be $S_{3} \times S_{1}$ with periodic boundary conditions in the (Euclidean) time. This choice is dictated by practical reasons. Geometric structures used to build simplicial manifolds of CDT are characterized by their position in spatial and time directions. In particular we use two types of four-simplices: $\{4,1\}$-simplices with four vertices at time $t$ and one at $t \pm 1$ and $\{3,2\}$-simplices with three vertices at $t$ and two at $t \pm 1$. All simplices of a particular type are assumed to have the same sizes. The discretized Regge action in this case takes a form [29-31]:

$$
S_{R}=-\left(K_{0}+6 \Delta\right) N_{0}+K_{4}\left(N^{\{4,1\}}+N^{\{3,2\}}\right)+\Delta N^{\{4,1\}}
$$

where $N_{0}$ is the total number of vertices in the triangulation, $N^{\{4,1\}}$ and $N^{\{3,2\}}$ are the total numbers of simplices of type $\{4,1\}$ and type $\{3,2\}$, respectively. $K_{0}, K_{4}$ and $\Delta$ are the (bare) dimensionless coupling constants obtained by the discretization of the continuous action (1.1). An additional geometric parameter is the length $t_{\text {tot }}$ of the periodic time axis.

In numerical simulations the total four-volume of the universe is kept fixed. In practice this restricts the number $N^{\{4,1\}}$ to fluctuate around a fixed value $\bar{N}_{41}$. For a fixed space-time topology the number of CDT triangulations with $\bar{N}_{41}\{4,1\}$ simplices grows exponentially with $\bar{N}_{41}$. This exponential growth determines the critical value $K_{4}^{\text {crit }}$ of the bare lattice cosmological constant $K_{4}$. Requiring the average $\left\langle N^{\{4,1\}}\right\rangle$ to be fixed is equivalent to fixing the bare cosmological constant $K_{4}$ to be close to the critical value $K_{4}^{\text {crit }}$. For $K_{4}<K_{4}^{\text {crit }}$ 


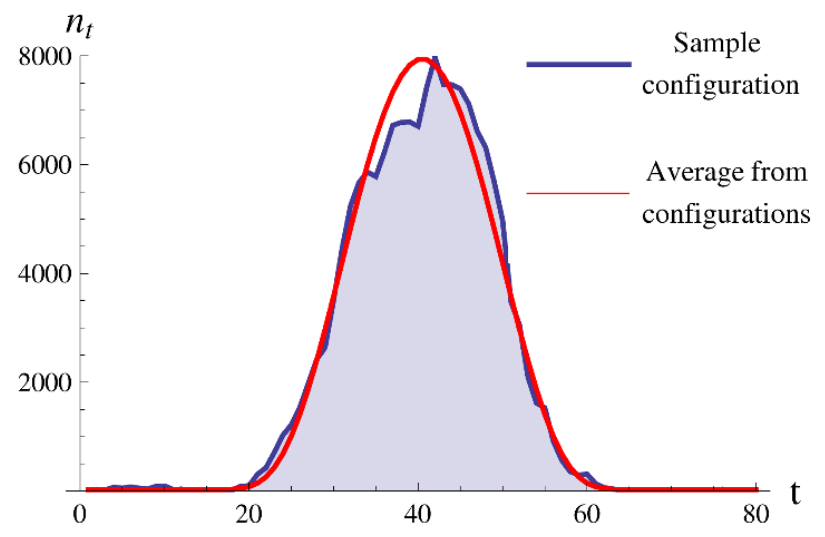

Figure 1. The measured distribution $n_{t}$ of a $\{4,1\}$ volume inside the de Sitter phase. The data are for coupling constants $K_{0}=2.2$ and $\Delta=0.6$. The blue line represents a single configuration generated in the Monte Carlo simulations. The red line represents the distribution, averaged over many configurations in a way described in [42].

the partition function

$$
\mathcal{Z}=\sum_{\mathcal{T}} e^{-S_{R}}
$$

becomes divergent.

The remaining two bare coupling constants can be chosen freely and the choice will determine the "physics" of the (lattice) theory. Numerical simulations proved that depending on these values the quantum system can be in three different phases [34,35]. From a physical point of view the most interesting is the de Sitter phase, where a typical geometry can be viewed as a quantum fluctuation around a semi-classical regular four-sphere, or rather four-ellipsoid with different scale in time and spatial directions [36-41]. A natural quantity used to parametrize the configurations is the distribution $n_{t}$ of $\{4,1\}$ volumes as a function of the discrete time $t . n_{t}$ is closely related to the scale factor in the minisuperspace approach to quantum gravity. $n_{t}$ is equal to twice the number of three-dimensional tetrahedra which form a spatial slice (with $S^{3}$ topology) at time $t$. This is because each spatial tetrahedron located in time-slice $t$ is shared by precisely two $\{4,1\}$ four-simplices, one with its fifth vertex at time-slice $t+1$ and one with its fifth vertex at time-slice $t-1$ (and of course both four-simplices have four vertices at time-slice $t$, namely the four vertices of the spatial tetrahedron they share). In the de Sitter phase the distribution of $n_{t}$ has a characteristic shape (figure 1), consisting of the blob which fluctuates around a four-sphere and a stalk with an almost minimal size. The stalk is present because the global topology $S_{3} \times S_{1}$ is not allowed to be broken in the computer simulations. The distribution of spatial volume can be averaged over many statistical independent configurations obtained in the numerical simulations. In the blob the average spatial volume profile $\left\langle n_{t}\right\rangle \propto \cos ^{3}(t / B)$ which corresponds to a (Wick rotated) de Sitter solution of Einstein's equations. Fluctuations around this semi-classical trajectory $\Delta n_{t}=n_{t}-\left\langle n_{t}\right\rangle$ are correlated for different $t$. The covariance matrix of fluctuations can be measured. The inverse of the covariance 
matrix can be used to determine the effective action in terms of $n_{t}$ [29-31, 42]. It was shown in [43] that it corresponds to a naively discretized minisuperspace action

$$
S_{\mathrm{eff}}^{\text {blob }}=\frac{1}{\Gamma} \sum_{t}\left(\frac{\left(n_{t}-n_{t+1}\right)^{2}}{n_{t}+n_{t+1}}-\lambda n_{t}+\mu n_{t}^{1 / 3}+\mathcal{O}\left(n_{t}^{-1 / 3}\right)\right),
$$

where $\Gamma$ is proportional to the effective Newton's constant while the effective cosmological constant $\lambda$ together with the parameter $\mu$ fix the total 4 -volume of the universe.

This form of the action leads in a natural way to a path-integral representation with the weight $\exp \left(-S_{\text {eff }}\right)$ of each configuration given by a product

$$
\exp \left(-S_{\text {eff }}\right) \equiv \prod_{t} \exp \left(-L_{\text {eff }}\left(n_{t}, n_{t+1}\right)\right)
$$

of pseudo-local transfer matrix elements

$$
\left\langle n_{t}|M| n_{t+1}\right\rangle \propto \exp \left(-L_{\text {eff }}\left(n_{t}, n_{t+1}\right)\right)
$$

linking neighbouring spatial slices. In this form all details of the geometric structure space at a given spatial slice are wiped out and we assume that it makes sense to use the effective quantum states $\left|n_{t}\right\rangle$ with unit norm as an eigenstate basis at each slice.

The transfer matrix can be measured in numerical simulations. In [44] we used this concept to determine the form of the effective action inside the de Sitter phase. We found that the symmetrized form of (1.4) with minor small-volume correction fits very well to our numerical data both in the blob and the stalk range of the CDT universe. This result was obtained in numerical simulations of systems with small time extension $\left(t_{\text {tot }}=2,3,4\right)$.

In CDT there exists a "genuine" transfer matrix $M_{\text {gen }}$ connecting states at time $t$ and time $t+1$. These states can be chosen as the states of spatial geometries, and in this approach a given spatial geometry is completely characterized be the corresponding $D T$ triangulation of $S^{3}$ (which is part of the $4 \mathrm{~d}$ CDT triangulation). Thus we have by definition for the "genuine" transfer matrix:

$$
\begin{aligned}
& \left\langle T(t+n)\left|M_{\text {gen }}^{n}\right| T(t)\right\rangle= \\
& \quad=\sum_{T(t+i), 1<i<n-1}\left\langle T(t+n)\left|M_{\text {gen }}\right| T(t+(n-1))\right\rangle \cdots\left\langle T(t+1)\left|M_{\text {gen }}\right| T(t)\right\rangle .
\end{aligned}
$$

The number of states $|T(t)\rangle$ is of course much larger than the number of so-called "effective" quantum states $\left|n_{t}\right\rangle$ mentioned above. The claim that the effective transfer matrix describes CDT well contains two aspects, namely

$$
\left\langle T(t+1)\left|M_{\text {gen }}\right| T(t)\right\rangle \sim\left\langle n_{t+1}|M| n_{t}\right\rangle,
$$

for generic states $|T\rangle$, as long as we only measure $n_{t}$, and even stronger

$$
\left\langle T(t+n)\left|M_{\text {gen }}^{n}\right| T(t)\right\rangle \sim\left\langle n_{t+n}\left|M^{n}\right| n_{t}\right\rangle,
$$

again when we only look at $n_{t}$. To be reassured that the effective transfer matrix approach is correct we have to check (1.9) for large $n$, that is for systems where $t_{\text {tot }} \gg 1$. The aim is 
to reproduce the full CDT results (the volume profile $\left\langle n_{t}\right\rangle$ and quantum fluctuations $\Delta n_{t}$ ) by studying the simplified effective model based on the measured transfer matrix.

We also want to extend our analysis of the effective transfer matrix/action to two other phases of CDT which are the analogues of the branched polymer and collapsed phases of DT. This is especially interesting in the context of phase transitions. Recent results $[45,46]$ showed that the phase transition between the de Sitter phase and the collapsed phase is a second (or higher) order phase transition. This makes it a natural candidate in the quest for UV fixed points of CDT. Therefore it is important to understand better the nature of the CDT phase transitions from a microscopic perspective.

\section{Methodology of the transfer matrix measurements}

In order to investigate the properties of CDT in four dimensions we have performed computer simulations of systems with (time) periodic boundary conditions and $S^{3}$ spatial topology. The action used in the computer simulations is the Regge discretization of the Einstein-Hilbert action, given by eq. (1.2).

Studies of the covariance matrix of spatial volume fluctuations,

$$
C_{t t^{\prime}} \equiv\left\langle\left(n_{t}-\left\langle n_{t}\right\rangle\right)\left(n_{t^{\prime}}-\left\langle n_{t^{\prime}}\right\rangle\right)\right\rangle
$$

suggest that the effective action couples only adjacent time slices and that there exists an effective transfer matrix, namely the one defined by eq. (1.6).

Inside the de Sitter phase (also called phase ' $\mathrm{C}$ ') the measurement and parametrization of the transfer matrix is straightforward. In the other two phases it has to be done with some care. The most problematic phase is the 'time collapsed' phase (also called phase 'B'), in which time translation symmetry is strongly broken in generic triangulations. Measurements inside phase ' $\mathrm{B}$ ' required modifying the Monte Carlo code used in earlier computer simulations. The new measurement method uses a system with just two time slices, and one has to avoid artificial repetition of (sub)simplices (the problem does not occur when the time direction has more than two time slices). We checked that inside the de Sitter phase the results of the new method are fully consistent with previous results based on the systems with 3 or 4 time slices.

In order to measure the transfer matrix we need systems with a small time period $t_{\text {tot }}$. In our transfer matrix parametrization the probability to measure the combination of 3 -volumes $n_{t}=N^{\{4,1\}}(t)$ in times $t=1 \ldots t_{\text {tot }}$ is given by:

$$
P^{\left(t_{\text {tot }}\right)}\left(n_{1}, n_{2}, \ldots, n_{t_{\text {tot }}}\right)=\frac{\left\langle n_{1}|M| n_{2}\right\rangle\left\langle n_{2}|M| n_{3}\right\rangle \ldots\left\langle n_{t_{\text {tot }}}|M| n_{1}\right\rangle}{\operatorname{tr} M^{t_{\text {tot }}}} .
$$

In a system with two time slices $\left(t_{\text {tot }}=2\right)$ we have:

$$
P^{(2)}\left(n_{1}, n_{2}\right)=\frac{\left\langle n_{1}|M| n_{2}\right\rangle\left\langle n_{2}|M| n_{1}\right\rangle}{\operatorname{tr} M^{2}},
$$

which implies:

$$
\langle n|M| m\rangle \propto \sqrt{P^{(2)}\left(n_{1}=n, n_{2}=m\right)},
$$


where we use the assumption that due to time-reflection symmetry the transfer matrix is also symmetric.

We can also use the probability distributions measured in Monte Carlo simulations with $t_{\text {tot }}=3$ and 4 (this was done in our earlier investigations). In this approach the transfer matrix elements can be computed as

$$
\langle n|M| m\rangle \propto \frac{P^{(3)}\left(n_{1}=n, n_{2}=m\right)}{\sqrt{P^{(4)}\left(n_{1}=n, n_{3}=m\right)}} .
$$

We checked that both approaches agree inside the de Sitter phase 'C'.

The advantage of the new method with $t_{\text {tot }}=2$ is twofold. First of all one needs only to measure a single probability distribution, thus leading to a reduction of computer time and to smaller statistical errors (since one does not need to combine two probability measurements like in (2.3)). However, more importantly, by an appropriate choice of volume fixing (see below) one can measure off-diagonal elements of the transfer matrix with much higher precision. It is especially important when extracting the kinetic part of the effective action in the ' $\mathrm{B}$ ' phase and in the third phase (which is called the ' $\mathrm{A}$ ' phase).

To perform the computer simulations efficiently one has to introduce some kind of volume fixing. This is done by adding to the usual Regge action (1.2) an additional volume fixing term:

$$
S_{R} \rightarrow S_{R}+S_{V F}
$$

In our simulations with $t_{\text {tot }}=2$ we use the global volume fixing ${ }^{1}$ either with a quadratic or a linear potential:

$$
S_{V F}=\epsilon\left(n_{1}+n_{2}-n_{v o l}\right)^{2} \quad \text { or } \quad S_{V F}=\epsilon\left|n_{1}+n_{2}-n_{v o l}\right| .
$$

The effect of the volume fixing term can be easily removed from the measured transfer matrix $\widetilde{M}$ defined by (2.2) by setting:

$$
\langle n|M| m\rangle=e^{\frac{1}{2} \epsilon\left(n+m-n_{v o l}\right)^{2}}\langle n|\widetilde{M}| m\rangle \text { or }\langle n|M| m\rangle=e^{\frac{1}{2} \epsilon\left|n+m-n_{v o l}\right|}\langle n|\widetilde{M}| m\rangle,
$$

for a quadratic or a linear volume fixing, respectively.

The volume fixing correction (2.5) clearly affects the diagonal elements of the transfer matrix used in the analysis of the the potential term (see below), whereas the cross-diagonal elements, important for the determination of the kinetic term, are simply rescaled for $n+m=$ const.

\section{The effective model in the de Sitter phase}

Recent results show that for small $t_{\text {tot }}$ the measured transfer matrix in the de Sitter phase does not depend on the number of slices supporting the decomposition (2.1). An example of the measured transfer matrix is plotted in figure 2 .

\footnotetext{
${ }^{1}$ Our previous approach used (2.3) based on probability distributions measured in systems with $t_{\text {tot }}=$ 3,4 , and we used a local volume fixing procedure (see [44] for details). The transfer matrix measurement with global volume fixing is possible only with $t_{\text {tot }}=2$ and is especially suitable in the 'A' and ' $\mathrm{B}$ ' phases where generic configurations typically have very different spatial volumes in neighbouring time slices.
} 


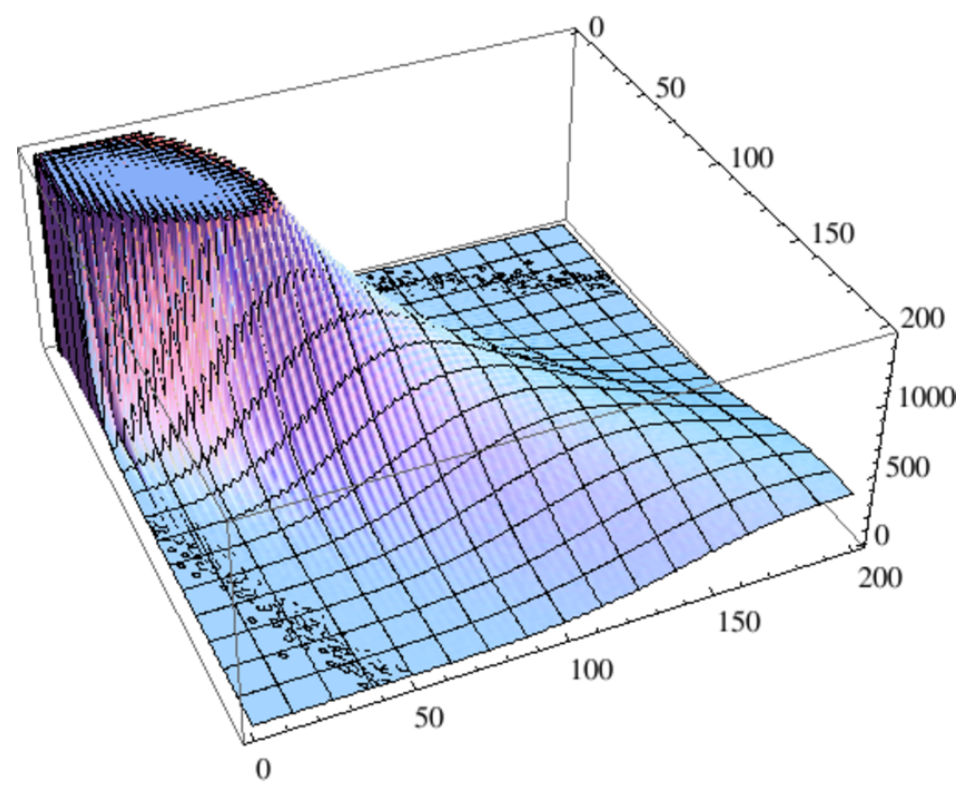

Figure 2. Measured transfer matrix elements $\langle n|M| m\rangle$ in the de Sitter phase 'C'. For small values of $n$ and $m$ we observe strong discretization effects. For larger volumes the behaviour is smooth.

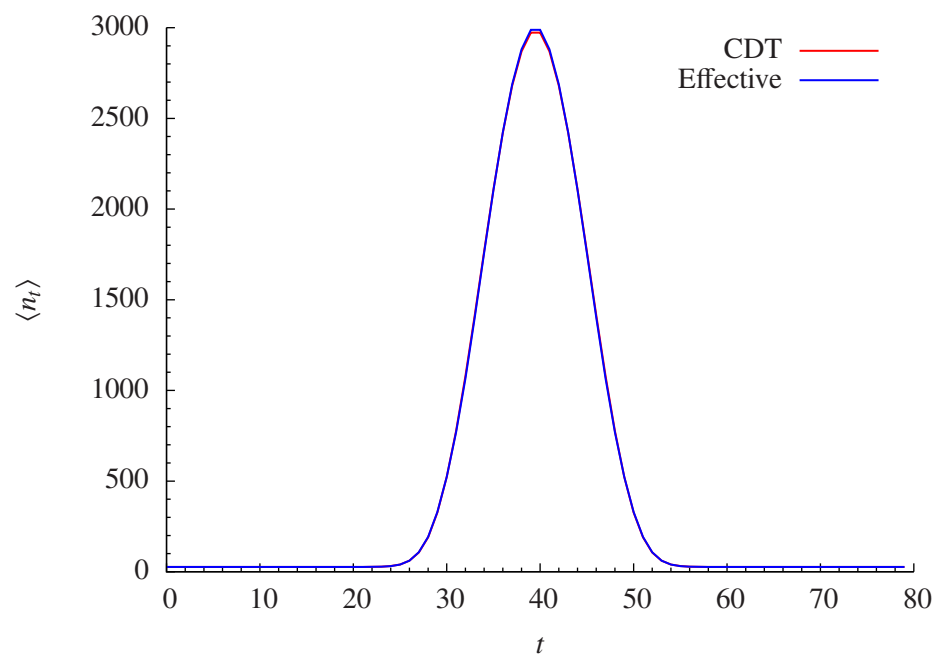

Figure 3. The average volume profile $\left\langle n_{t}\right\rangle$ for full CDT in the de Sitter phase (red) and the effective model (blue) for $t_{\text {tot }}=80$ and $\bar{N}_{41}=40 \mathrm{k}$.

In order to reconstruct the results of the full CDT simulations we will need matrix elements $M_{n m} \equiv\langle n|M| m\rangle$ for large volumes $n, m$. As can be seen from figure 3, for a total four-volume with $\bar{N}_{41}=40 \mathrm{k}$ the largest spatial volumes $n_{t}$ reach values above 3000 . Technically it is difficult to measure matrix elements in such wide range, but we can use extrapolation for large volumes.

For small volumes the transfer matrix elements are dominated by very strong discretization effects as can be seen in figure 2, but as $n$ and $m$ increase the behaviour becomes much smoother. For sufficiently large spatial volumes the transfer matrix is very well described 

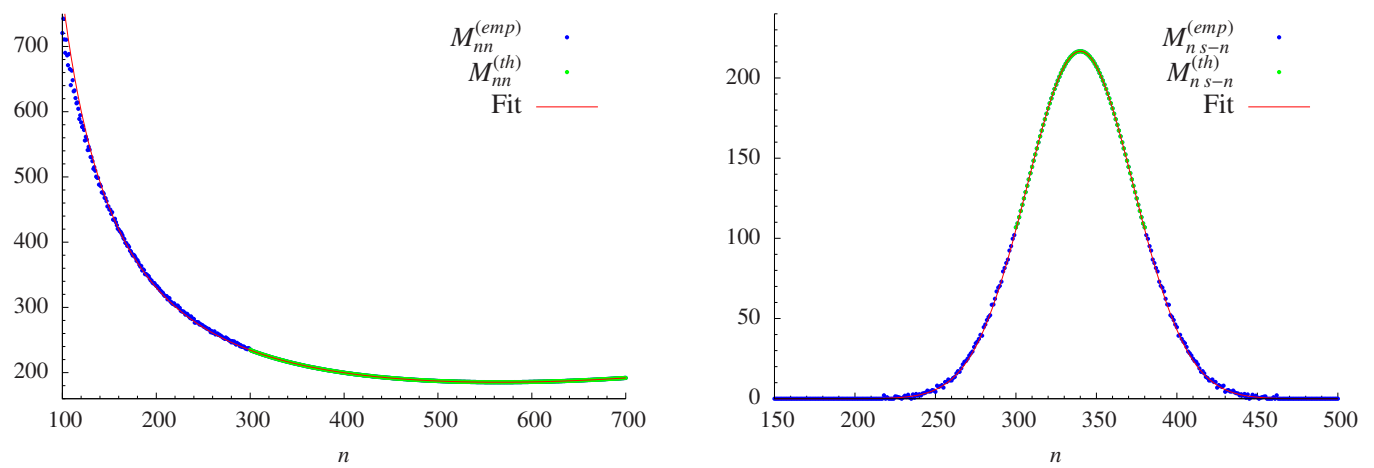

Figure 4. The effective transfer matrix in the de Sitter phase, merged from the empirical matrix (blue) and theoretical matrix (green). The theoretical matrix (red line) is determined by a best fit to the empirical matrix in an overlap region where $n$ and $m$ are in the range 250-700 as described in the text. Left: diagonal $\langle n|M| n\rangle$. Right: anti-diagonal $\langle n|M| s-n\rangle, s=680$.

by the effective Lagrangian introduced in [44],

$$
\begin{gathered}
L_{\mathrm{eff}}(n, m)= \\
=\frac{1}{\Gamma}\left[\frac{(n-m)^{2}}{n+m-2 n_{0}}+\mu\left(\frac{n+m}{2}\right)^{1 / 3}-\lambda\left(\frac{n+m}{2}\right)-\delta\left(\frac{n+m}{2}\right)^{-\rho}\right] .
\end{gathered}
$$

We use the measured (empirical) transfer matrix $M_{n m}^{(\mathrm{emp})}, 250<n, m<700$, to determine the parameters $\Gamma, n_{0}, \mu, \lambda, \delta$ and $\rho$, by making a best fit of

$$
M_{n m}^{(\mathrm{th})}=\mathcal{N} e^{-L_{\mathrm{eff}}(n, m)},
$$

to $M_{n m}^{(\mathrm{emp})}$. For larger values of $n, m$ we then use $M_{n m}^{(\mathrm{th})}$, with $L_{\mathrm{eff}}(n, m)$ determined by this fit.

Finally, we thus define the semi-empirical transfer matrix by

$$
M_{n m}=\left\{\begin{array}{l}
M_{n m}^{(\mathrm{emp})} n<t h r \text { or } m<t h r \\
M_{n m}^{(\mathrm{th})} \text { otherwise }
\end{array}\right.
$$

where $t h r$ is a threshold $(t h r=300)$. When one of the entries is smaller than the threshold we use the measured matrix elements. When both entries are larger than the threshold we use the extrapolating function (3.2). Figure 4 presents the diagonal $\langle n|M| n\rangle$ (left) and an anti-diagonal $\langle n|M| s-n\rangle$ (right) of the semi-empirical transfer matrix. The blue points denote the empirical part of $M$, used for volumes below the threshold, i.e. for $n<300$ or $m<300$. The red line presents a theoretical fit of the form (3.2), the fitting range being $250-700$. The green points correspond to the theoretical part of $M$ given by (3.2) for volumes above the threshold, i.e. for $n$ and $m$ larger than 300. The agreement between the empirical transfer matrix and the fit (plotted as the red line) is very good also below $n=250$ but gets even better above the threshold. The extrapolation (3.2) allows us to expand the transfer matrix to volumes which are not easily accessible by 


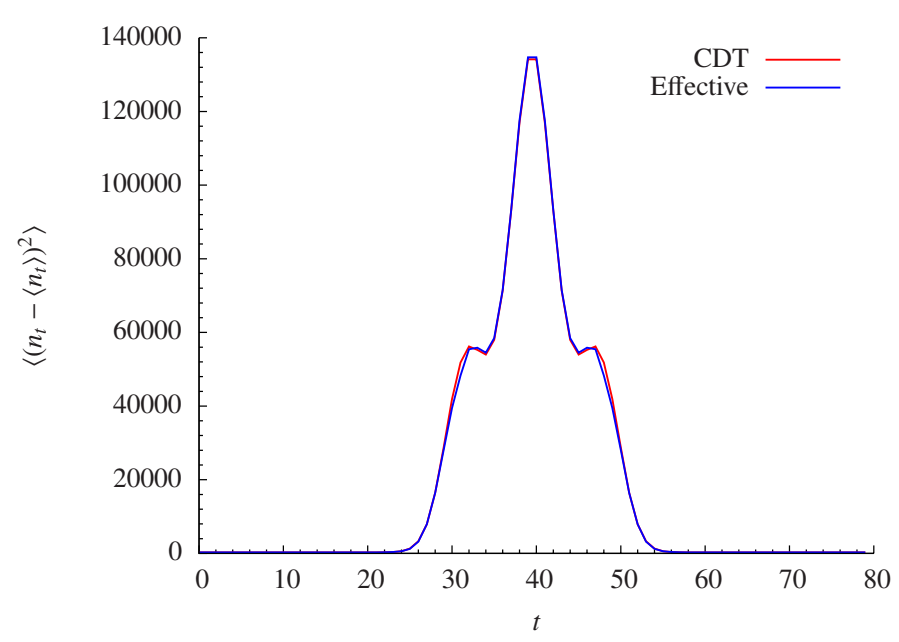

Figure 5. Variance of spatial volumes $\left\langle\left(n_{t}-\left\langle n_{t}\right\rangle\right)^{2}\right\rangle$ for full CDT in the de Sitter phase (red line) and the effective model (blue line). It corresponds to the diagonal of the covariance matrix $C_{t t^{\prime}}$.

direct measurement. Nevertheless, because the effective Lagrangian describes perfectly the measured transfer matrix in the range where we can make the comparison, and seemingly gets better with increasing values of the entries $(n, m)$, this extrapolation beyond actual empirical data should not be of any importance when judging the validity of the effective transfer matrix decomposition (2.1).

We now introduce an effective model which aims to reproduce results of the full CDT model in the de Sitter phase. In this approach configurations are given by volume profiles $\left\{n_{t}\right\}$ rather than by triangulations $\mathcal{T}$. The model is based on the effective transfer matrix decomposition (2.1) with the usual total volume fixing term used in the full CDT simulations:

$$
P\left(n_{1}, \ldots, n_{t_{\mathrm{tot}}}\right) \propto\left\langle n_{1}|M| n_{2}\right\rangle\left\langle n_{2}|M| n_{3}\right\rangle \cdots\left\langle n_{t_{\mathrm{tot}}}|M| n_{1}\right\rangle e^{-\epsilon\left(\sum_{t} n_{t}-\bar{N}_{41}\right)^{2}} .
$$

We can specify the probability distribution of configurations using the transfer matrix $\langle n|M| m\rangle$ constructed in (3.3). In order to recover results of the original model we have to access matrix elements for large volumes.

Next, we apply standard Monte Carlo methods to generate the configurations, i.e.

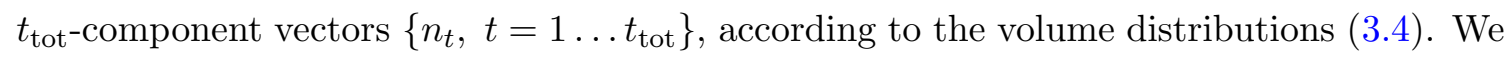
use the same number of slices $t_{\text {tot }}$ and total volume $\bar{N}_{41}$ as in the full CDT simulations. As before, we measure the average volume profile $\left\langle n_{t}\right\rangle$ and the covariance matrix $C_{t t^{\prime}} \equiv$ $\left\langle\left(n_{t}-\left\langle n_{t}\right\rangle\right)\left(n_{t^{\prime}}-\left\langle n_{t^{\prime}}\right\rangle\right)\right\rangle$. The results obtained using this effective model are almost identical to the results obtained with the original, full CDT model when we are well into phase ' $\mathrm{C}$ ' (the modification of eq. (3.1) needed in phase ' $\mathrm{A}$ ' and ' $\mathrm{B}$ ' will be discussed in section 5 and section 6 ). Figure 3 shows the average volume profile $\left\langle n_{t}\right\rangle$ measured in the full CDT simulations (the red line) and in the reduced model simulations (the blue line). The two curves overlap almost exactly. The diagonal of the covariance matrix $C_{t t}$, i.e. variance of $n_{t}$, is shown in figure 5. Again, results of the original, full CDT model (the red line) and of the effective model (the blue line) are in complete agreement. 


\section{Extracting the kinetic and potential terms}

The transfer matrix measured in computer simulations can be used to determine the form of the effective action/Lagrangian. It was shown in [44] that in the de Sitter phase ' $\mathrm{C}$ ' the action is approximated very well by a simple discretization of the continuum minisuperspace action with a minor small-volume correction, more precisely by the $L_{\mathrm{eff}}(m, n)$ given in (3.1). The form of the discretization suggests that the transfer matrix can be factorized into a potential and a kinetic part:

$$
\langle n|M| m\rangle=\mathcal{N} \underbrace{\exp (-v[n+m])}_{\text {potential }} \underbrace{\exp \left(-\frac{(n-m)^{2}}{k[n+m]}\right)}_{\text {kinetic }},
$$

where the functions:

$$
\begin{aligned}
v[n+m] & =\frac{\mu}{\Gamma}\left(\frac{n+m}{2}\right)^{1 / 3}-\frac{\lambda}{\Gamma}\left(\frac{n+m}{2}\right)-\frac{\delta}{\Gamma}\left(\frac{n+m}{2}\right)^{-\rho} \\
k[n+m] & =\Gamma \cdot\left(n+m-2 n_{0}\right),
\end{aligned}
$$

will be called the potential and kinetic coefficients, respectively.

The potential part can be easily analyzed by looking at the diagonal elements of the transfer matrix:

$$
v[2 n]=-\log \langle n|M| n\rangle+\log \mathcal{N},
$$

while the kinetic term requires extracting the cross-diagonal elements:

$$
\langle n|M| s-n\rangle=\mathcal{N}(s) \exp \left(-\frac{(2 n-s)^{2}}{k[s]}\right) .
$$

By measuring the potential coefficient for different $n$ and the kinetic coefficient for different $s$ one verifies that eqs. (4.2) and (4.3) hold in the de Sitter phase [44].

We will apply the same factorization to analyze the measured transfer matrices in phases 'A' and 'B'. Further, we will check how the kinetic and potential terms change when we move between phase ' $\mathrm{A}$ ' and ' $\mathrm{C}$ ', as well as between phase ' $\mathrm{B}$ ' and ' $\mathrm{C}$ '.

\section{The transfer matrix in phase ' $A$ '}

Phase ' $\mathrm{A}$ ' is separated from phase ' $\mathrm{C}$ ' by a first order phase transition, which we meet if we start in phase ' $\mathrm{C}$ ' and increase the coupling constant $K_{0}$ (see figure 17 ).

We measured the transfer matrix in a generic point inside phase 'A' $\left(K_{0}=5.0, \Delta=\right.$ $\left.0.4, K_{4}=1.22\right)$ using the method described in section 2 with $t_{\text {tot }}=2$ and a quadratic volume fixing term.

The kinetic part can be analyzed by looking at cross-diagonal elements of the transfer matrix: $\langle n|M| m\rangle=\langle n|M| s-n\rangle$. The generic shape of the measured cross-diagonal is presented in figure 6 where $\langle n|M| m\rangle$ is plotted as a function of $(n-m)=d$. The shape looks very different from the typical behaviour in phase ' $C$ ' (where it is Gaussian, cf. 


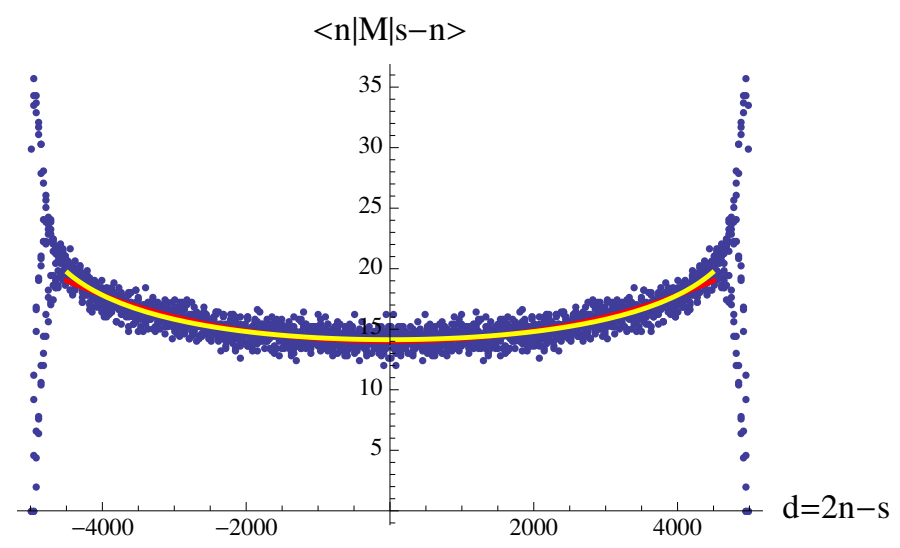

Figure 6. Sample cross-diagonal of the measured transfer matrix inside phase 'A' $\left(K_{0}=5.0, \Delta=\right.$ $\left.0.4, K_{4}=1.22\right)$. The data are for $s=n+m=5000$. The red line corresponds to the fit of the 'artificial' "anti-Gaussian" (5.1). The yellow line is the best fit to the the effective Lagrangian (5.6).

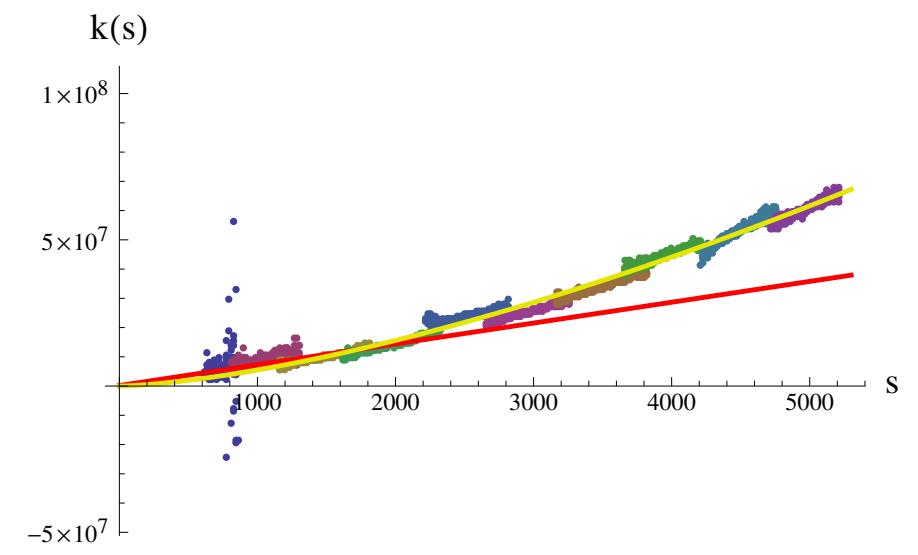

Figure 7. The kinetic coefficient $k[s]$ measured in phase 'A' $\left(K_{0}=5.0, \Delta=0.4, K_{4}=1.22\right) . k[s]$ is not linear (red line) but can be fitted using the function defined by eq. (5.2) (yellow line).

figure 4). Disregarding strong discretization effects for small volumes one could naively say that the cross-diagonals of the measured transfer matrix can be fitted with a very flat "anti-Gaussian" function. Indeed we tried to fit (red line in figure 6):

$$
\langle n|M| s-n\rangle=\mathcal{N}(s) \exp \left(\frac{d^{2}}{k[s]}\right) .
$$

The kinetic coefficient $k[s]$ as a function of $s$ is presented in figure 7 . In contrast to the behaviour in phase ' $\mathrm{C}$ ', $k[s]$ is no longer linear. It can be fitted with the following parametrization (yellow curve in figure 7 )

$$
k[s]=k_{0} s^{2-\alpha} .
$$

The best fit is for $\alpha=0.50 \pm 0.01$ and $k_{0}=175 \pm 10$.

The "anti-Gaussian" behaviour of the kinetic part is somewhat strange. Covariance analysis of triangulations in phase ' $\mathrm{A}$ ' shows that the volume distributions in different time 
slices are not correlated. Therefore the kinetic part should vanish and what we observe may just be an artifact of the measurement/parametrization method.

Let us assume that in phase ' $\mathrm{A}$ ' the 3 -volume distributions at different time slices are independent, but that we have a local potential term. This leads naturally to an effective Lagrangian of the form:

$$
L_{A}(n, m)=\mu\left(n^{\alpha}+m^{\alpha}\right)-\lambda(n+m) .
$$

One can change this parametrization to: $s=n+m, d=n-m$ and assume $d / s$ small. $^{2}$ Then we obtain

$$
\begin{aligned}
L_{A}(n, m) & =\mu\left(\frac{s}{2}\right)^{\alpha}\left[\left(1+\frac{d}{s}\right)^{\alpha}+\left(1-\frac{d}{s}\right)^{\alpha}\right]-\lambda s \\
& =-\lambda s+\mu\left(\frac{s}{2}\right)^{\alpha}\left[2+\alpha(\alpha-1)\left(\frac{d}{s}\right)^{2}\right]+\mathcal{O}\left(d^{4}\right) .
\end{aligned}
$$

For $\alpha<1$ we effectively get an "anti-Gaussian" behaviour of the transfer matrix crossdiagonals (5.1) with

$$
k[s]=\frac{2^{\alpha}}{\mu \alpha(1-\alpha)} s^{2-\alpha},
$$

exactly in line with our measurements (5.2). From the fitted values of $\alpha$ and $k_{0}$ one can calculate $\mu=0.032 \pm 0.002$.

As a check of parametrization (5.3) one may use the effective Lagrangian $L_{A}$ to fit cross-diagonal elements of the measured transfer matrix:

$$
\langle n|M| s-n\rangle=\mathcal{N} \exp \left[-L_{A}(n, s-n)\right]=\mathcal{N}(s) \exp \left[-\mu n^{\alpha}-\mu(s-n)^{\alpha}\right] .
$$

The best fit for $\alpha=0.5$ is presented as a yellow curve in figure 6 and gives $\mu=0.022 \pm 0.001$. The parameter $\mu$ fitted for different cross-diagonals (as a function of $s$ ) is presented in figure 8. The value of $\mu$ tends to a constant for large volumes (big $s$ ) as discretization effects get smaller. The red line corresponds to $\mu=0.024$.

The analysis of the potential part is now straightforward:

$$
\log \langle n|M| n\rangle=-L_{A}(n, n)+\log \mathcal{N}=-2 \mu n^{\alpha}+2 \lambda n+\log \mathcal{N} .
$$

The diagonal elements of the measured transfer matrix together with the best fit of $\mu=$ 0.026 (for $\alpha=0.5$ ) are presented in figure 9 .

As a side remark we may go back to the analysis of the de Sitter phase 'C'. In this phase we use the parametrization (3.1) with symmetrized potential terms. As a result the potential coefficient is a function of the sum of volumes in the adjacent time slices $(v[n+m])$. If instead the true potential was not symmetric (a function of $n$ and $m$ separately) one should expect the same kind of effective "anti-Gaussian" term to appear. However this effect is very small compared to the generic Gaussian behaviour of the kinetic part. As a result, the kinetic coefficients $k[n+m]$ would be slightly modified, which may explain the existence of the non-vanishing but very small $n_{0}$ in the measured effective Lagrangian (3.1).

\footnotetext{
${ }^{2}$ For cross-diagonal terms it is not always true, as $d / s$ can be of order 1 and higher order corrections should be taken into account.
} 


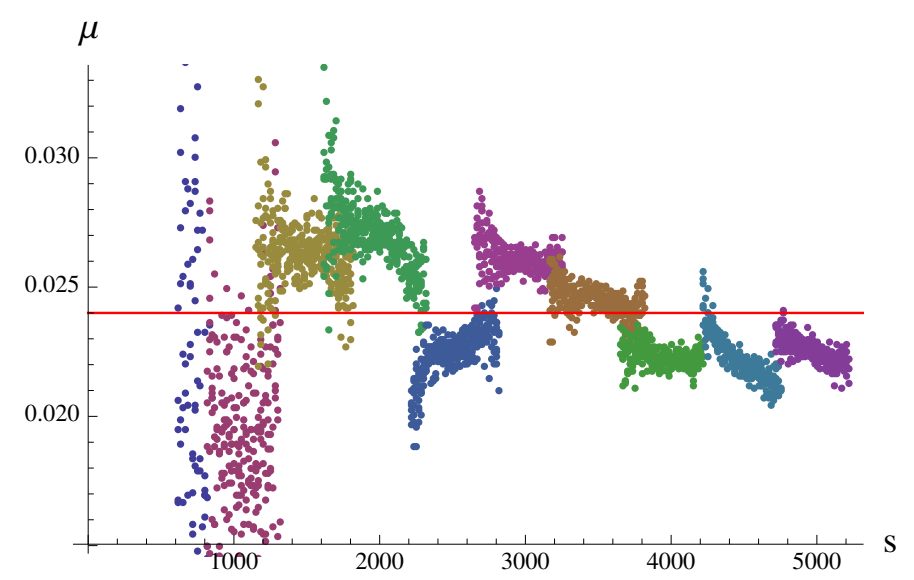

Figure 8. $\mu$ as a function of $s$ measured in phase 'A' $\left(K_{0}=5.0, \Delta=0.4, K_{4}=1.22\right)$. The value of $\mu$ stabilizes around 0.024 (the red line) as discretization effects vanish.

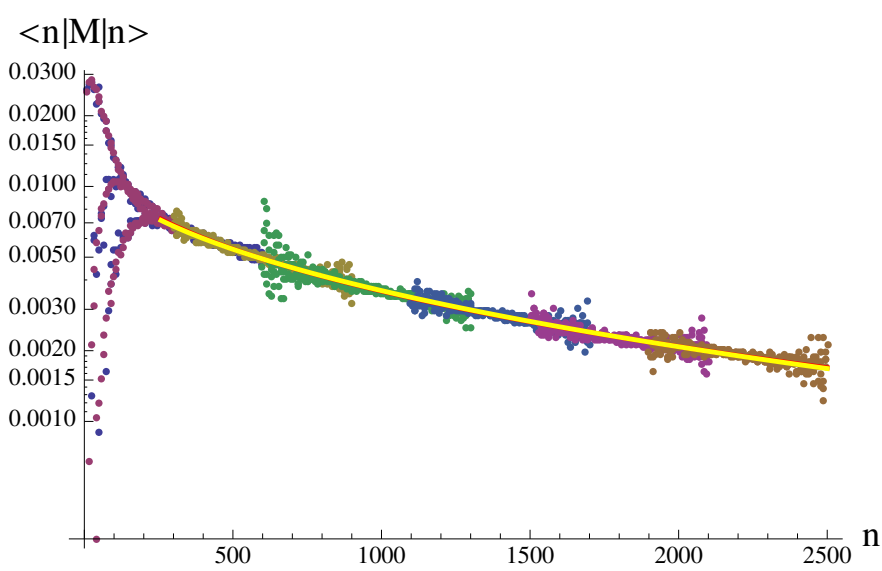

Figure 9. The diagonal elements of the transfer matrix measured in phase 'A' $\left(K_{0}=5.0, \Delta=\right.$ $0.4, K_{4}=1.22$ ) shown together with the best fit to eq. (5.7). The fit disregards strong discretization effects visible for small volumes.

\section{The transfer matrix in phase ' $\mathrm{B}$ '}

Starting at the generic point in phase ' $\mathrm{C}$ ' we reach phase ' $\mathrm{B}$ ' by decreasing $\Delta$ (see figure 17). Phase ' $\mathrm{C}$ ' and phase ' $\mathrm{B}$ ' are separated by a second or higher order phase transition.

The analysis of the transfer matrix in phase ' $\mathrm{B}$ ' is not straightforward. Generic triangulations in this phase are 'collapsed' i.e. the spatial 3 -simplices of almost all $\{4,1\}$ simplices are concentrated in a single time slice. As a result we do not have much information about volume-volume correlations.

As an additional issue, the potential part of the effective action inside phase ' $\mathrm{B}$ ' seems to suffer from a strong non-linear dependence on the total volume $s$ in the small to medium volume regime. In our simulations we fix the $K_{0}$ and $\Delta$ coupling constants of the Regge action (1.2), while $K_{4}$ (which is conjugate to the total four-volume of the triangulation) is fine-tuned to offset the spontaneously emerging entropic / potential term coming from the exponentially large number of configurations with constant total volume $s$. If the emerging 


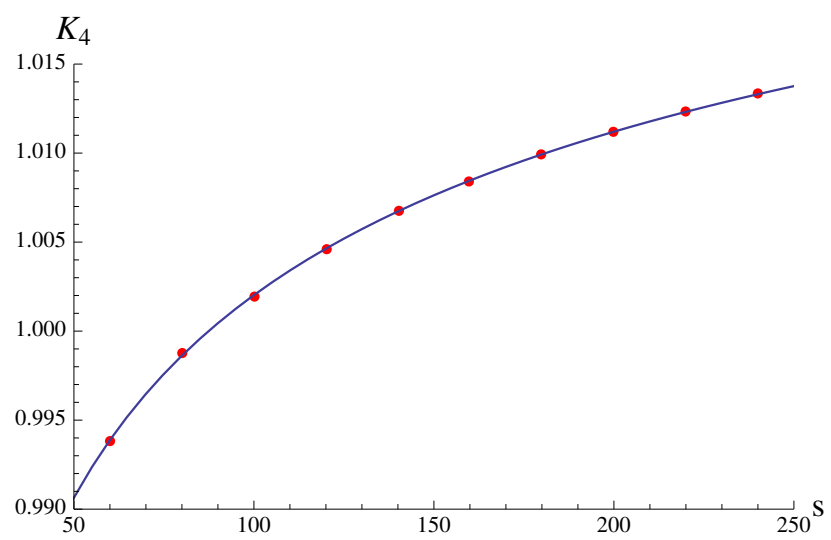

Figure 10. $K_{4}$ scaling with total volume ( $\mathrm{s}$ in '000) inside phase 'B' (for $K_{0}=2.2, \Delta=0.0$ ) and the best fit of eq. (6.1).

effective potential is linear (this is the exponentially growing number of configurations with $s$ ) the fine-tuned value of $K_{4}$ will be constant, independent of $s$. The non-linear corrections to the effective potential reflect sub-leading corrections to the exponentially growing number of configurations. They might be small power-like corrections which can effectively be neglected. Our simulations show that corrections to $K_{4}$ due to non-linear components in the effective action (3.1) in phase ' $\mathrm{C}$ ' and in the effective action (5.3) in phase 'A' indeed are negligible, even in the small volume region (they change the fourth significant digit, which is of the same order as the accuracy of the $K_{4}$ fine-tuning). The situation is much different in the ' $\mathrm{B}$ ' phase where the fine-tuned value of $K_{4}$ is strongly volume dependent even for relatively large volumes. It is illustrated in figure 10, where the value of $K_{4}$ is plotted as a function of total volume $s$ together with the fit:

$$
K_{4}(s)=K_{4}^{\infty}-\beta s^{-\gamma}
$$

The strong volume dependence of $K_{4}$ on $s$ implies that it is technically impossible to measure the transfer matrix in phase 'B' for the values of $K_{4}$ appropriate for a large volume limit. If we fix the $K_{4}$ value to the critical value corresponding to a large volume, effectively this value is "too large" and the system will oscillate around the minimally allowed configuration and only very seldom make detours to the large values of $s$ corresponding to the chosen value of $K_{4}$. To circumvent this problem we decided to use lower values of $K_{4}$ and analyze how a change of $K_{4}$ affects the measured transfer matrix. As a result we can (at least qualitatively) estimate the properties of the transfer matrix in the continuum limit.

In this section we present the results for measurement performed at a generic point in phase 'B' $\left(K_{0}=2.2\right.$ and $\left.\Delta=0.0\right)$. We start our analysis with the transfer matrix measured for $K_{4}=0.943$ using $t_{\text {tot }}=2$ with quadratic total volume fixing. We explicitly symmetrize the data: $\langle n|M| m\rangle=\langle m|M| n\rangle$ even though the measured probabilities of volume distributions are highly asymmetric in general. This is equivalent to regaining the time-reflection symmetry of the transfer matrix which is strongly broken by generic configurations. 

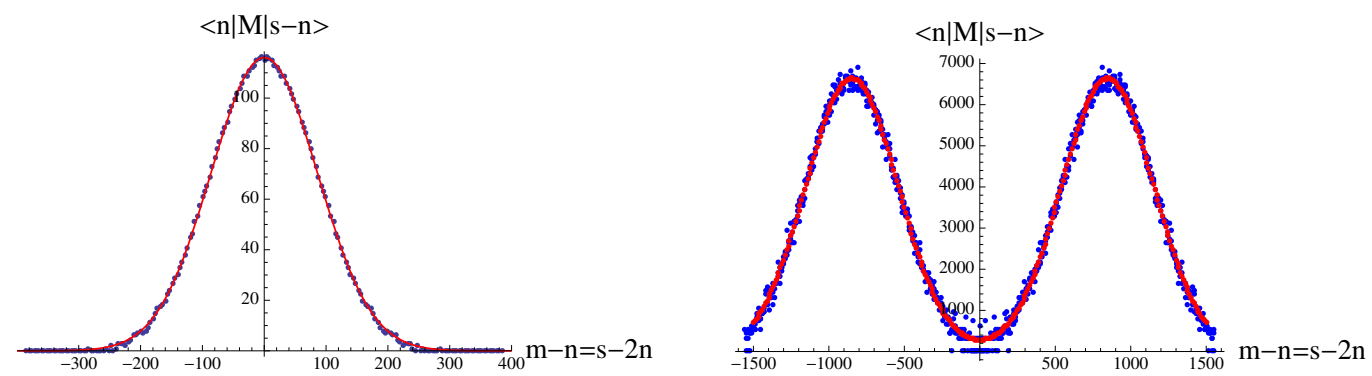

Figure 11. Cross diagonals of the transfer matrix measured in phase ' $\mathrm{B}$ ' (for $K_{0}=2.2, \Delta=0.0$ and $\left.K_{4}=0.943\right)$. The left chart shows the data below bifurcation point $\left(s=n+m<s^{b}\right)$. The right chart presents cross-diagonals above bifurcation point $\left(s>s^{b}\right)$. The best fits of eq. (6.2) are presented as red lines.

The typical behaviour of the cross-diagonal (kinetic) part of the measured transfer matrix $\langle n|M| m\rangle=\langle n|M| s-n\rangle$ strongly depends on $s=n+m$. For $s<s^{b}$ it looks the same as in phase ' $\mathrm{C}$ ' and can be well fitted with a single Gaussian (4.5) - see figure 11 (left). For $s>s^{b}$ the cross-diagonals split into the sum of two "shifted" Gaussians - see figure 11 (right). The value of the shift depends on $s=n+m$ (figure 12). All together the kinetic part can be parametrized by:

$$
\begin{aligned}
\langle n|M| m\rangle & =\langle n|M| s-n\rangle \\
& =\mathcal{N}(s)\left[\exp \left(-\frac{((m-n)-c[s])^{2}}{k[s]}\right)+\exp \left(-\frac{((m-n)+c[s])^{2}}{k[s]}\right)\right],
\end{aligned}
$$

where: $c[s]$ is (close to) zero for $s<s^{b}$ and (almost) linear for $s>s^{b}$ :

$$
c[s] \approx \max \left[0, c_{0}\left(s-s^{b}\right)\right] .
$$

This type of parametrization fits the measured data quite well (the red line in figure 12) and is convenient for our further analysis. We will call $s^{b}$ the bifurcation point. For our generic data $\left(K_{0}=2.2, \Delta=0\right.$ and $\left.K_{4}=0.943\right)$ the best fits yield: $s^{b}=2020$ and $c_{0}=0.31$.

Another phenomenological parametrization, which fits the data around bifurcation point even better (yellow curve in figure 12) is:

$$
c[s]=c_{0} s \exp \left(-s^{b} / s\right) .
$$

It is consistent with (6.3) for small and large $s$ (compared to $s^{b}$ ). We will return to this parametrization when analyzing the phase transitions in the next sections.

The last function that should be fitted is $k[s]$ which is very well approximated by a linear function, independently of whether we are below or above the bifurcation point (figure 13). The behaviour is consequently the same as in phase 'C', i.e. (4.3):

$$
k[s]=\Gamma\left(s-2 n_{0}\right) .
$$

The best fit yields: $\Gamma=36.8, n_{0}=5.4$ which is of the same order as the values measured in the de Sitter phase 'C'. 


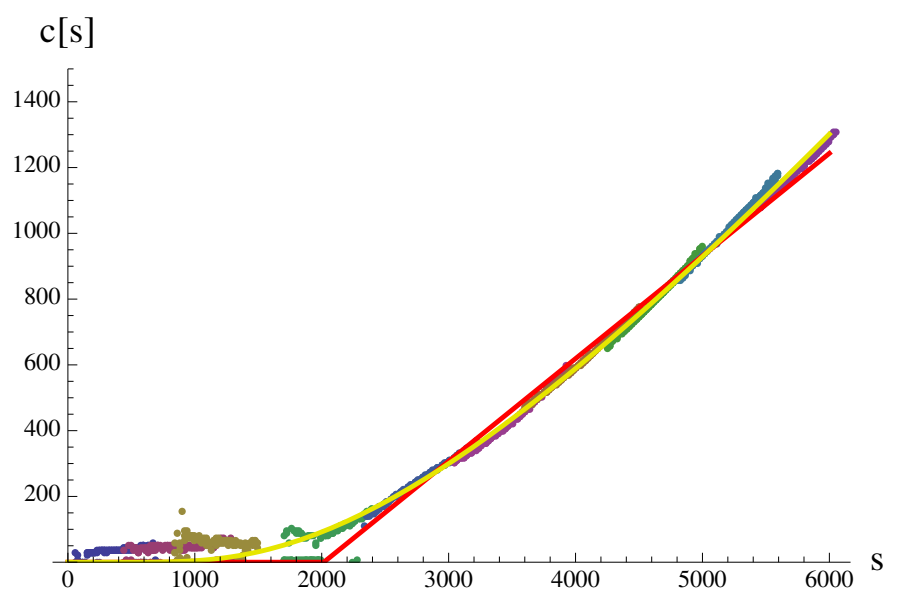

Figure 12. The bifurcation shift c $[\mathrm{s}]$ measured in phase 'B' (for $K_{0}=2.2, \Delta=0.0$ and $K_{4}=0.943$ ) together with the best fits using eq. (6.3) (red line) and (6.4) (yellow line).

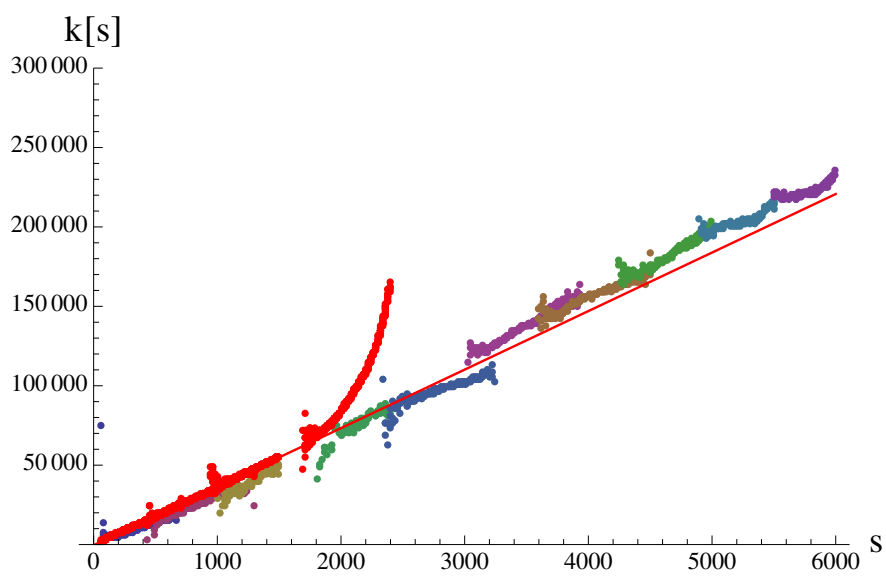

Figure 13. The kinetic coefficient $\mathrm{k}[\mathrm{s}]$ measured in phase 'B' (for $K_{0}=2.2, \Delta=0.0$ and $K_{4}=$ 0.943). The red points correspond to the fit of a single Gaussian (which is not valid after crossing bifurcation point (for $s=n+m>s^{b}$ )). Different colours correspond to the fit of two Gaussians (6.2) which is reliable also above the bifurcation point (for $s>s^{b}$ ). The bifurcation point $s^{b}$ can be identified as the point at which the fit of the single Gaussian starts to diverge from linear behaviour. It is consistent with the measured value of $s^{b}$ as presented in figure 12 .

As we are interested in properties of the transfer matrix in the large volume limit (where critical values of $K_{4}$ are much higher) it is important to check how the results depend on $K_{4}$. The plots of $c[s]$ and $k[s]$ for different $K_{4}$ are presented in figure 14. In general, the functional form of Eq's (6.2)-(6.5) is adequate for different values of $K_{4}$. With regards to the parameters entering in Eq's (6.2)-(6.5), the change of $K_{4}$ does not influence the position of the bifurcation point $s^{b}$, while the bifurcation slope $c_{0}$ and the effective Newton's constant $\Gamma$ rise as $K_{4}$ is increased.

Let us use these results to explain (at least qualitatively) the behaviour of the system in phase 'B'. In our 'full CDT' simulations (with $t_{\text {tot }}=80$ ) we analyze systems with large total volume ( $\geq 40 \mathrm{k}$ simplices) for which $K_{4}$ is big. As a result $c_{0}$, and consequently $c[s]$, 

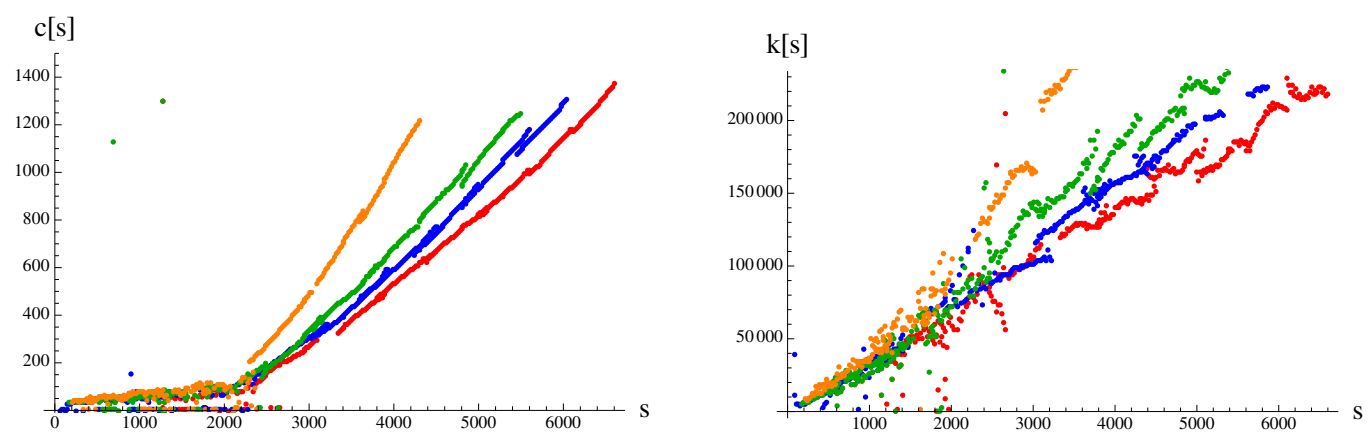

Figure 14. The bifurcation shift $c[s]$ (left) and the kinetic coefficient $k[s]$ (right) measured in phase 'B' $\left(K_{0}=2.2, \Delta=0.0\right)$ for different values of $K_{4}=0.933$ (red), 0.943 (blue), 0.953 (green), 0.973 (orange). The bifurcation point $s^{b}$ is stable whereas the slope of the shift $c_{0}$ and the value of $\Gamma$ rise as $K_{4}$ is increased.

are large in the interesting region $\left(s>s^{b}\right)$. Naively speaking, configurations with very large difference of spatial volume in the adjacent time slices should be favoured (most probable $(m-n)$ is large) and a kind of 'anti-ferromagnetic' behaviour with a ...-'large'-'small''large'-'small'-... . volume distribution observed. This is exactly what we see in CDT systems with small time periods $t_{\text {tot }}=2,4,6$ used in the transfer matrix measurements, but for $t_{\text {tot }}=80$ the observed behaviour is very different and the volume distribution is 'collapsed' to just one time slice. In order to explain this phenomena we must take into account the entropic factor (the potential part in the transfer matrix 'language'). Due to strong dependence of $K_{4}$ on the total volume, the exact measurement of the potential in the large volume range is beyond our reach at the moment. Instead let us present a theoretical model in which the potential is exactly the same as in phase ' $\mathrm{C}$ ', i.e. given by eq. (4.2). For simplicity we will consider only the leading behaviour by setting $\lambda, \delta, n_{0}=0$. Consequently:

$$
\begin{array}{r}
\langle n|M| m\rangle=\exp \left(-\frac{\mu}{\Gamma}\left(\frac{n+m}{2}\right)^{1 / 3}\right)\left[\exp \left(-\frac{\left((m-n)-\left[c_{0}\left(n+m-s^{b}\right)\right]_{+}\right)^{2}}{\Gamma(n+m)}\right)\right. \\
\left.+\exp \left(-\frac{\left((m-n)+\left[c_{0}\left(n+m-s^{b}\right)\right]_{+}\right)^{2}}{\Gamma(n+m)}\right)\right],
\end{array}
$$

where: $[.]_{+}=\max (., 0)$.

Now we can perform the same kind of 'effective' Monte Carlo simulations as explained in section 3 in which the theoretical transfer matrix (6.6) will be used to generate volume distributions $\left\{n_{t}, t=1 \ldots t_{\text {tot }}\right\}$ with the probability given by eq. (3.4). We set the parameters of our model to the values measured in the real 'full CDT' simulations: $\Gamma=37, \mu=15, s^{b}=2000$ and $c_{0}=0.1-0.3$. The resulting volume distribution for small and large $t_{\text {tot }}$ is presented in figure 15 and figure 16, respectively. As a reference case we also plot the volume distribution for $c_{0}=0$, for which we recover the generic behaviour found in the de Sitter phase ' $\mathrm{C}$ '.

For small $t_{\text {tot }}$ the expected 'anti-ferromagnetic' structure is observed, while for large $t_{\text {tot }}$ a single 'collapsed' blob forms. The strength of the 'collapsed' behaviour depends on $c_{0}$. 


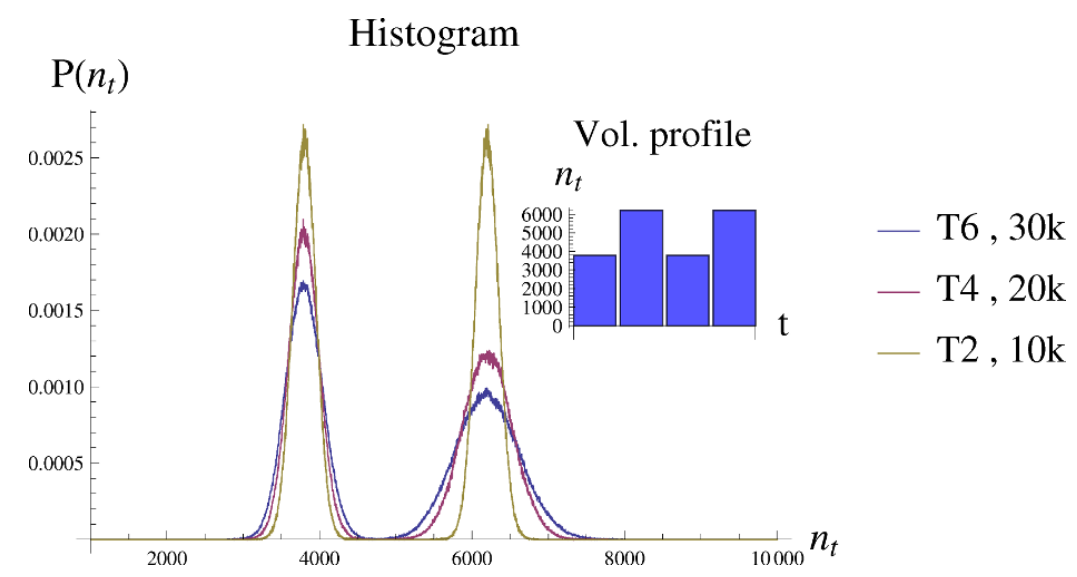

Figure 15. The histogram of the spatial volume distributions and the volume profile measured in the effective Monte Carlo model (6.6) for $c_{0}=0.3$ and $t_{\text {tot }}=2,4,6$. The two Gaussian peaks correspond to odd and even time slices, respectively. As a result the average volume profile is 'anti-ferromagnetic' with quantum fluctuations around: ...-3.8k-6.2k-3.8k-6.2k-...

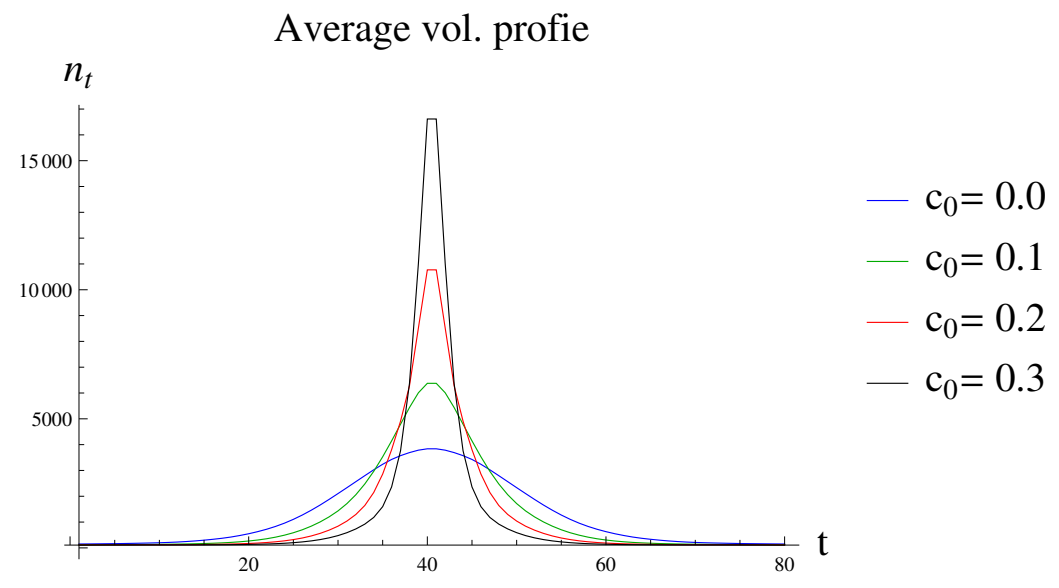

Figure 16. The average spatial volume measured in the effective Monte Carlo model (6.6) for $t_{\text {tot }}=80, \bar{N}_{41}=100 \mathrm{k}$ and different values of $c_{0}$. The shape of the volume profile is consistent with the 'collapsed' blob structure for $c_{0}>0$.

This simple model explains very well (at least at a qualitative level) the volume distribution inside phase ' $\mathrm{B}$ '. In reality we should take into account that the value of $c_{0}$ appropriate for large $K_{4}$ used in 'full CDT' simulations is probably much bigger (leading to a much more narrow distribution for large $t_{\text {tot }}$ ). In addition the actual entropic/potential part present in the full CDT model may corroborate the idea of a 'narrowing' of the volume distribution compared to the one we observe in the toy model defined by (6.6).

\section{Phase transitions}

When one applies conventional methods to analyze the phase transitions observed in fourdimensional CDT one obtains strong evidence that the 'A'- C' transition is a first order transition while the ' $\mathrm{B}$ '- ' $\mathrm{C}$ ' transition is a second (or higher) order transition [45, 46]. These 


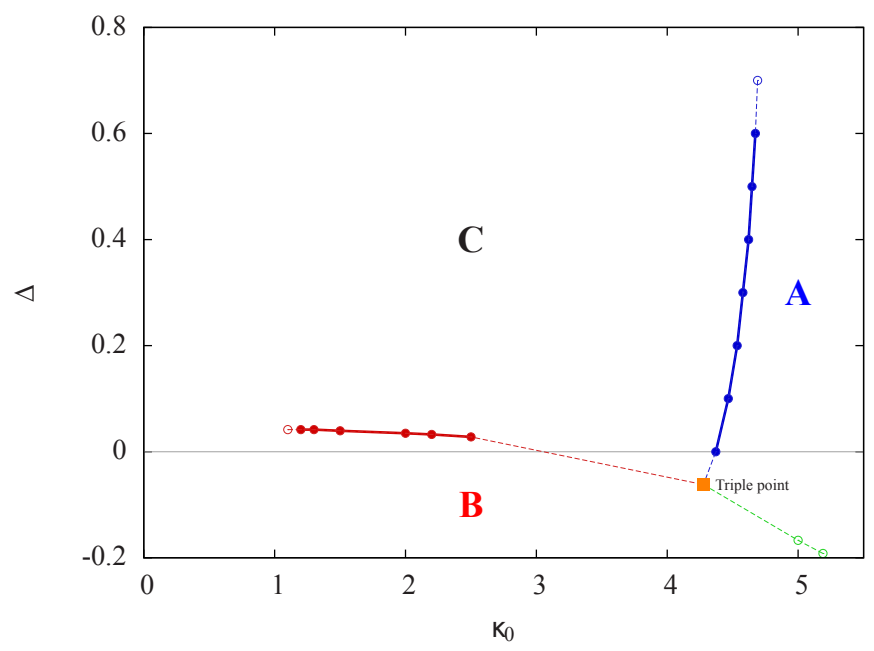

Figure 17. The CDT phase diagram measured by 'traditional' methods based on the order parameter analysis.

results are based on the analysis of order parameters defined as variables conjugate to bare coupling constants in the Regge action (1.2). By looking at the susceptibility of the order parameters one can identify the position of the phase transition lines in the phase diagram (figure 17). At the same time the critical exponents, scaling properties and large volume behaviour enable one to analyze the order of the phase transition. The order parameters in question reflect some global characteristics of the CDT triangulations (e.g. the ratio $N_{0} / N^{\{4,1\}}$. A change in such order parameters does not necessarily give much insight into the 'microscopic' nature of the phase transitions, which is an obvious drawback of this approach. In particular, if one wants to find algorithms which can beat the critical slowing down observed near the transitions. We will try to use the transfer matrix to obtain additional information about the phase transitions.

The 'A'-' $\mathrm{C}$ ' phase transition is easily visible in the kinetic part of the transfer matrix. When we approach the 'A'- $\mathrm{C}$ ' phase transition line from phase ' $\mathrm{C}$ ' (by increasing $K_{0}$ and keeping $\Delta$ fixed) the kinetic part of the minisuperspace effective action (3.1) vanishes smoothly. Near an 'A'-'C' phase transition point the cross-diagonals of the measured transfer matrix are almost constant. Just after the phase transition we can observe the formation of the 'artificial' anti-Gaussian term discussed in detail in section 4 . For $\Delta=0.6$ the phase transition point can be identified at $K_{0}=4.75 \pm 0.05$ (see figure 18) which is fully consistent with the location found using the 'traditional' approach used in $[45,46]$.

The 'B'-'C' phase transition is not as easily visible. In the previous section we parametrized the kinetic part of the transfer matrix in phase ' $\mathrm{B}$ ' by a sum of two Gaussians (6.2)-(6.5). An obvious parameter to look at is the bifurcation point $s^{b}$. For small volumes $s=n+m<s^{b}$ the kinetic part is the same as in phase ' $\mathrm{C}$ '. The difference, responsible for the 'collapsed' behaviour in phase ' $\mathrm{B}$ ', is observed for large volumes $s>s^{b}$. Thus, it is a natural conjecture that the ' $\mathrm{B}$ '- ' $\mathrm{C}$ ' phase transition is related to the appearance of a bifurcation point $s^{b}$. However, as we will see that is not the case. If we start in phase 'B', keep $K_{0}$ fixed and increase $\Delta$ in order to cross the 'B'-'C' phase transition line the value of $s^{b}$ also increases. Thus, it is natural to treat the condition $s^{b} \rightarrow \infty$ as a sign of a 

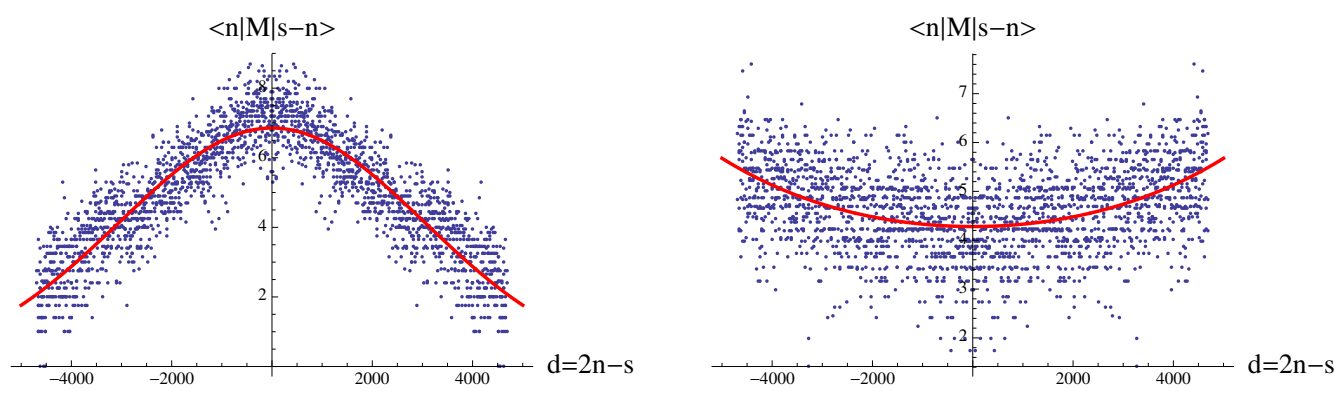

Figure 18. The cross-diagonal elements of the transfer matrix for $s=n+m=5000$ measured for $\Delta=0.6$. The left chart presents data for $K_{0}=4.7$ (phase ' $\mathrm{C}$ ') while the right chart presents the data for $K_{0}=4.8$ (phase 'A'). The change of the behaviour is clearly visible, which enables us to identify the phase transition point.

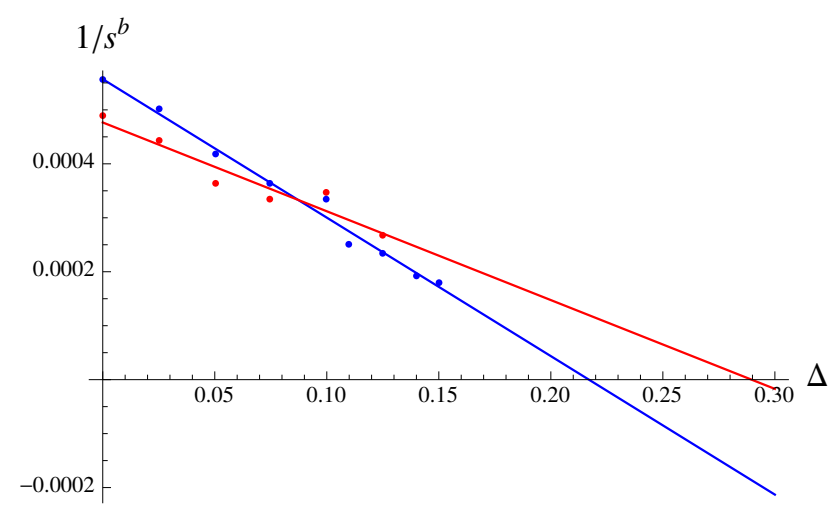

Figure 19. The (inverse of) bifurcation point $s^{b}$ as a function of $\Delta$ (for fixed $K_{0}=2.2$ ). The colours correspond to two different ways of extracting $s^{b}$ : by direct measurements (red points) and indirectly, as the values of $s$ where a single Gaussian does not fit the distributions (blue points) see footnote. ${ }^{3}$

phase transition. In figure 19 we present the plot of $1 / s^{b}$ as a function of $\Delta$ for $K_{0}=2.2$. Different colours correspond to two methods of measuring $s^{b}$. The relation seems to be linear, implying the transition occurs for $\Delta=0.2-0.3$. This value of $\Delta$ is much higher than critical value measured in the 'traditional' approach $(\Delta \approx 0.05)$. By using $s^{b}$ as an indicator of a phase transition we are seemingly observing something different from the formerly observed 'B'-'C' transition. We will discuss this in the next section.

\section{A new 'bifurcation' phase?}

In the previous section we provided evidence that a new kind of transition occurs when we start out in phase ' $\mathrm{B}$ ' and increase $\Delta$. It is related to the disappearance of the bifurcation

\footnotetext{
${ }^{3}$ Red points correspond to $s^{b}$ determined by fitting eq. (6.3) to the measured data. This method requires performing transfer matrix measurements also in the region of volumes much higher then $s^{b}$ which is difficult as we approach the phase transition point. Blue points correspond to the indirect determination of $s^{b}$, identified as the point at which a single Gaussian does no longer fit the measured cross-diagonals (recall figure 13 for details). The larger the $\Delta$ the more difficult it is to observe the shift away from a singleGaussian distribution. Therefore the values of $1 / s^{b}$ for large $\Delta$ are probably underestimated when using this second method.
} 


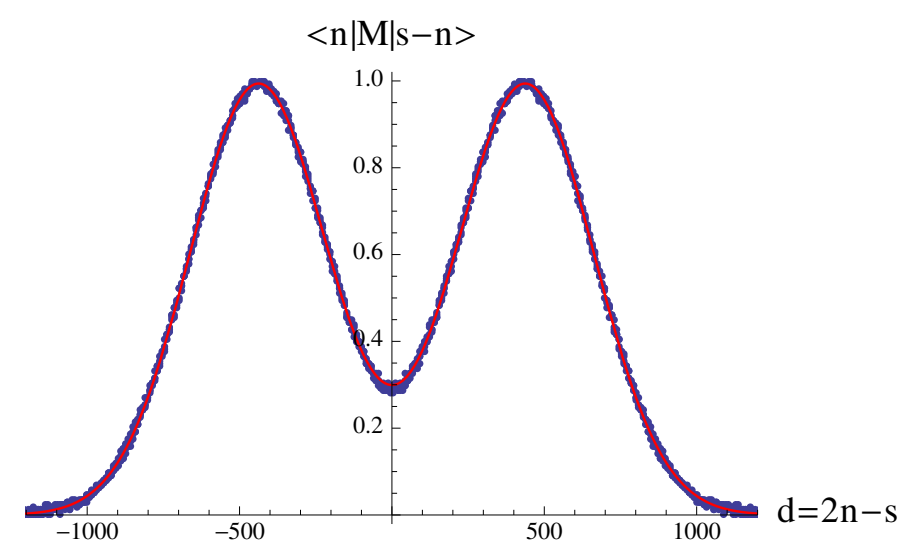

Figure 20. The cross diagonal elements of the transfer matrix measured for $K_{0}=2.2$ and $\Delta=$ 0.125 which according to the 'traditional' approach lies well inside the de Sitter phase ' $\mathrm{C}$ '. The data are measured for $s=n+m=15000$. The double-Gaussian bifurcation structure characteristic for phase ' $\mathrm{B}$ ' configurations is still clearly visible.

point $s^{b}$ observed in phase ' $\mathrm{B}$ ' in the kinetic term. However, this disappearance of $s^{b}$ is observed for the values of $\Delta$ much larger than the $\Delta$-value where the 'conventional' 'B'- 'C' phase transition is located. It is thus located in the region of coupling constant space we conventionally have denoted phase ' $\mathrm{C}$ '. The position of the new transition point is based on the interpolation of the bifurcation point $s^{b}$ to infinity as a function of $\Delta$. One may argue that this relation may change in the vicinity of the transition, lowering the "critical' $\Delta$ value. However, it is possible to observe the 'bifurcation' structure also for $0.1<\Delta<0.3$ (i.e. in the region of the ' $\mathrm{C}$ ' phase bordering the conventional 'B'-' $\mathrm{C}$ ' phase transition) if one uses a total volume $\bar{N}_{41}$ large enough (see figure 20) and performs the simulations with small $t_{\text {tot }}$. For the same values of $\Delta$ the average volume profile for large $t_{\text {tot }}$ has the typical blob-shape characteristic for the de Sitter phase 'C'. In fact, if one looks at the transfer matrix data nothing special happens while crossing the conventional ' $\mathrm{B}$ '- $\mathrm{C}$ ' phase transition line $\left(\Delta \approx 0.05\right.$ for $\left.K_{0}=2.2\right)$. This is in obvious contradiction with the 'traditional' phase diagram presented in figure 17.

To explain this phenomenon we refer back to figure 15 (right) where the average volume profiles for the effective Monte Carlo model with the 'bifurcated' transfer matrix (6.6) were shown. For small bifurcation slopes $c_{0}$ the volume profiles are practically identical with those observed in the generic de Sitter phase. This is true even for systems with large total volumes $\left(\bar{N}_{41}=100 \mathrm{k}\right)$. For medium bifurcation slopes the volume profile contracts in the time direction, but the general shape does not change much. Only for large $c_{0}$ does one observe something which resembles a 'collapse' of the blob in the time-direction.

If one plots the average volume profiles measured in 'full CDT' for $K_{0}=2.2$ and different values of $\Delta=0.0-0.6$ (figure 21) the result looks qualitatively the same. For $\Delta \geq 0.4$ the shape does not change much. An increasing contraction in the time-diction takes place for $0.1 \leq \Delta \leq 0.3$. Finally, crossing the 'conventional' 'B'-'C' phase transition at $\Delta=0.05$ we observe the 'collapse' of the blob in the time direction, characteristic of a generic phase 'B' configuration. 


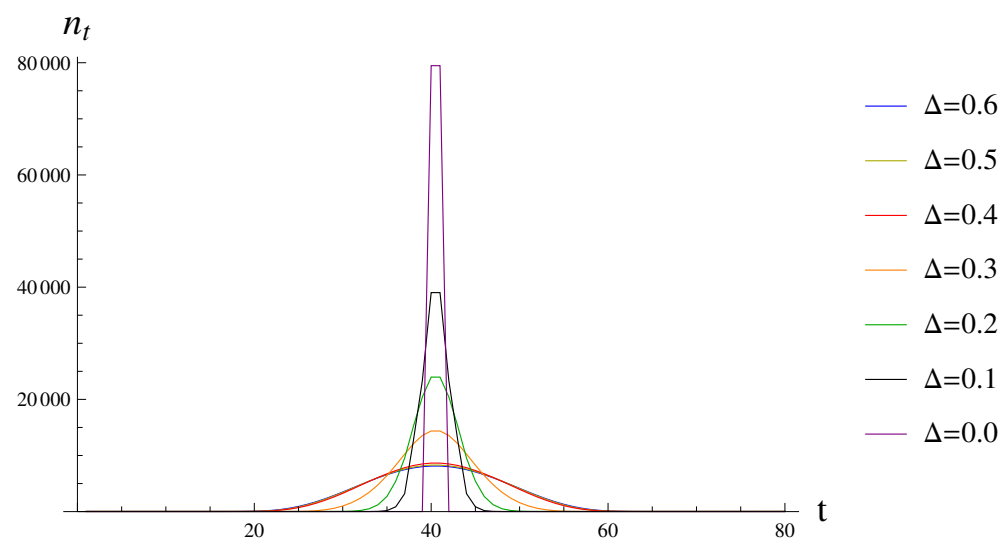

Figure 21. The average spatial volume profiles measured in 'full CDT' for $K_{0}=2.2, t_{\text {tot }}=80$ and $\bar{N}_{41}=160 \mathrm{k}$.

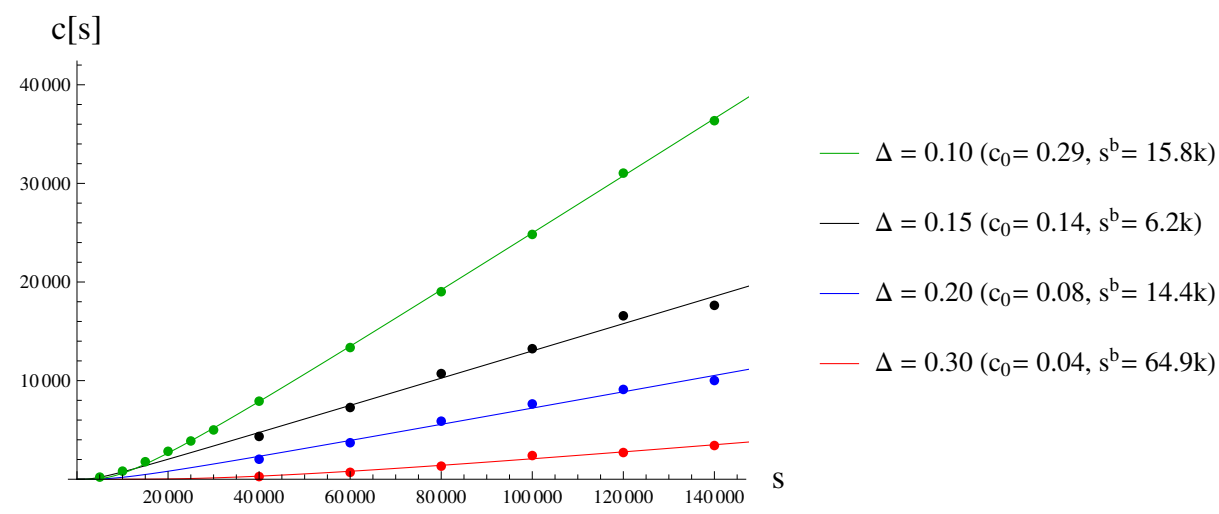

Figure 22. The bifurcation shifts (dots) measured for $K_{0}=2.2$ and for different values of $\Delta$, and the best fits of $c[s]=c_{0} \exp \left(-s^{b} / s\right) s$ to these data (lines).

To justify this picture we measured the bifurcation structure for $0.1 \leq \Delta \leq 0.3$. It required using large total volumes which substantially increased the computer simulation time. To get better statistics we focused on selected cross-diagonals of the measured transfer matrix by choosing the global volume fixing term (2.4) very peaked at $n_{v o l}=20 \mathrm{k}, 40 \mathrm{k}$, $60 \mathrm{k}, 80 \mathrm{k}, \ldots$ and by performing measurement only if $n+m=n_{v o l}$. We fitted the doubleGaussian (6.2) to the measured cross-diagonals and extracted the bifurcation shift $c\left[n_{v o l}\right]$. The parametrization (6.4) seems to reproduce our data best, and we used it to calculate the values of the bifurcation point $s^{b}$ and the slope $c_{0}$. We present these results in figure 22 . As expected the bifurcation slope $c_{0}$ gradually grows when $\Delta$ is decreased. This results in the observed contraction of the time-extent of the blob. The gradual disappearance of the double peak structure with increasing $\Delta$ might also apply to other functions of the triangulations, and that might explain why for instance the 'traditional' order parameter used to identify the 'B'- 'C' transition is seemingly insensitive to the new 'bifurcation' transition we observe around $\Delta=0.3-0.4$ for $K_{0}=2.2$.

Summarizing, we conclude that the new 'bifurcation' phase may exist in fourdimensional CDT. This phase should lie between the ' $\mathrm{B}$ ' and ' $\mathrm{C}$ ' phases. Its generic 

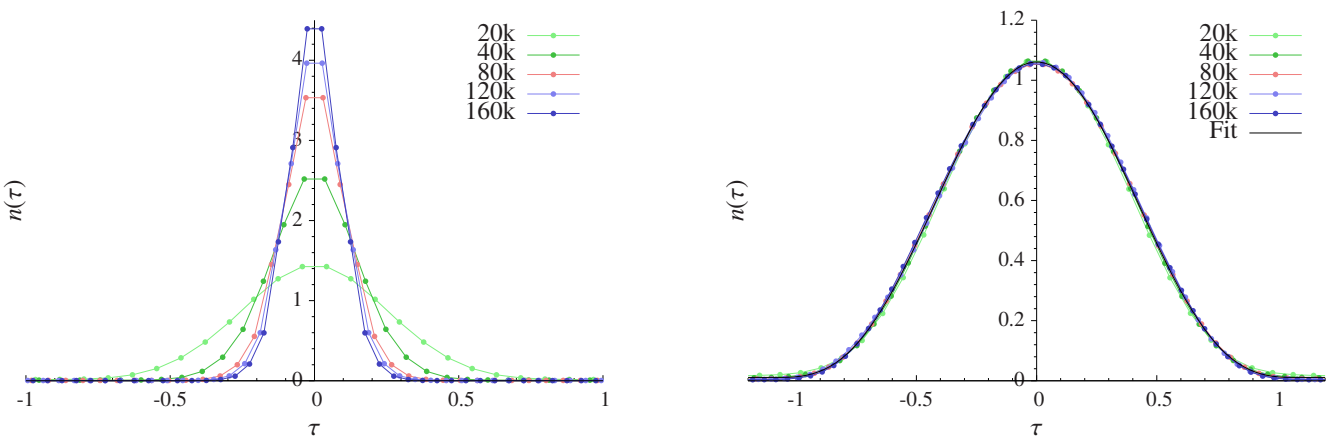

Figure 23. The CDT distributions of spatial volumes $n_{t}$ measured for different total four-volumes $\bar{N}_{41}$. The left figure shows the distributions in the 'bifurcation' phase $\left(K_{0}=2.2, \Delta=0.125\right)$, and the right figure the distributions in the Sitter phase ' $\mathrm{C}$ ' $\left(K_{0}=2.2, \Delta=0.6\right)$. Different colours correspond to different total volumes $\bar{N}_{41}$. The data were rescaled, according to: $\tau=t / \bar{N}_{41}^{1 / d_{H}}$ and $n(\tau)=n_{t} / \bar{N}_{41}^{1-1 / d_{H}}$, to fit a single curve, assuming the Hausdorff dimension $d_{H}=4$. The lack of scaling in the new phase is an important difference compared to the generic phase ' $\mathrm{C}$ '.

3-volume (temporal) distribution measured in 'full-CDT' with large $t_{\text {tot }}$ has blob structures resembling those found in phase ' $\mathrm{C}$ ', but there seem to be important differences. As an example we present the spatial volume distributions for different total volumes $\left(\bar{N}_{41}\right)$ measured inside the new phase for $K_{0}=2.2$ and $\Delta=0.125$ (figure 23). The volume distributions were rescaled both in time $\left(\tau=t / \bar{N}_{41}^{1 / d_{H}}\right)$ and space $\left(n(\tau)=n_{t} / \bar{N}_{41}^{1-1 / d_{H}}\right)$ with $d_{H}=4$. In generic phase ' $\mathrm{C}$ ' $(\Delta=0.6)$ the Hausdorff dimension $d_{H}=4$, and the rescaled volume distributions fall onto a universal curve. The scaling for $\Delta=0.125$ is evidently different. This is a strong argument in favour of the existence of a genuinely new phase. In fact the scaling (or rather lack of scaling) of volume profiles in the bifurcation region indicates that $d_{H} \rightarrow \infty$ for large volumes. This is consistent with our effective transfer matrix model (6.6) and is characteristic of the generic phase ' $\mathrm{B}$ ' - see figure 24. However, there is also no reason to doubt that the 'traditional' ' $\mathrm{B}$ '- ' $\mathrm{C}$ ' transition is still there, so seemingly we have discovered a new phase separating the 'old' phase ' $\mathrm{C}$ ' and phase ' $\mathrm{B}$ '.

\section{$9 \quad$ Summary and conclusions}

The recently introduced effective transfer matrix labeled only by the spatial volume [44] provides an interesting tool for analyzing Causal Dynamical Triangulations in four dimensions. Using Monte Carlo simulations of the complete CDT theory and the factorization (2.1), we have determined the effective transfer matrix.

Assuming validity of decomposition (2.1) we introduced an effective transfer matrix model reducing the degrees of freedom only to spatial volumes, i.e. a model which neglects completely the internal structure of slices. The simplified model reproduces perfectly all results obtained so far using the full model of four-dimensional CDT.

In phase ' $\mathrm{C}$ ' the effective Lagrangian

$$
L_{C}(n, m)=\frac{1}{\Gamma}\left[\frac{(n-m)^{2}}{n+m-2 n_{0}}+\mu\left(\frac{n+m}{2}\right)^{1 / 3}-\lambda\left(\frac{n+m}{2}\right)-\delta\left(\frac{n+m}{2}\right)^{-\rho}\right]
$$



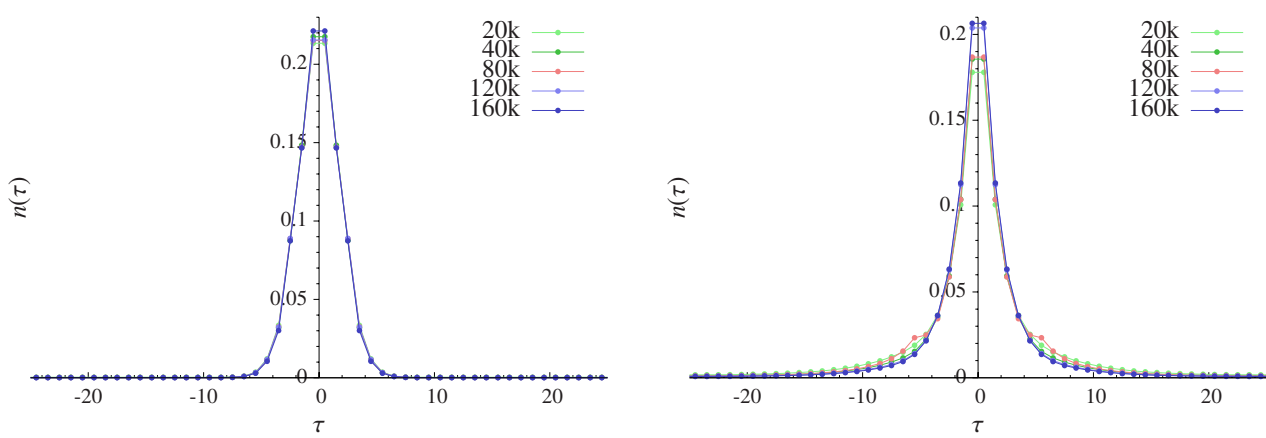

Figure 24. The left figure shows CDT distributions of spatial volumes $n_{t}$ measured in the 'bifurcation' phase $\left(K_{0}=2.2, \Delta=0.125\right)$. The right figure shows the $n_{t}$ distributions generated from the 'effective' Monte Carlo model (6.6) with bifurcation slope $c_{0}=0.3$. Different colours correspond to different total volumes $\bar{N}_{41}$. The data were rescaled, according to: $\tau=t / \bar{N}_{41}^{1 / d_{H}}$ and $n(\tau)=n_{t} / \bar{N}_{41}^{1-1 / d_{H}}$, to fit a single curve, assuming the Hausdorff dimension $d_{H}=\infty$.

describes very well the measured transfer matrix and can seemingly be used also for large volumes.

Our present investigations were partly inspired by a recent study [47] which used the effective Lagrangian

$$
L_{\text {eff }}=c_{1} \frac{2(n-m)^{2}}{n+m}+c_{2} \frac{m^{1 / 3}+n^{1 / 3}}{2}
$$

to define an effective one-dimensional transfer matrix. The authors studied the phase diagram as a function of the coupling constants $c_{1}$ and $c_{2}$. Interestingly, they observed a phase diagram which was quite similar to the CDT phase diagram. Clearly the effective Lagrangian (9.2) resembles the more precisely determined Lagrangian (9.1) which can be used to represent the full CDT model in phase ' $\mathrm{C}$ '. Thus we wanted to check, using the full CDT model, if (9.2) could really be viewed as giving a precise description of the full CDT model in phase 'A' and ' $\mathrm{B}$ ' for certain choices of $c_{1}$ and $c_{2}$. It turned out that this was not really the case.

We provide strong evidence that inside the 'uncorrelated' phase 'A' the effective Lagrangian takes the form

$$
L_{A}(n, m)=\mu\left(n^{\alpha}+m^{\alpha}\right)-\lambda(n+m),
$$

with $\alpha \neq 1 / 3$. The absence of a kinetic term can be interpreted as a causal disconnection of different time slices, i.e. the phenomenon of 'asymptotic silence' observed both in classical and quantum approaches to gravity in the regime of extreme curvatures/energy densities [48]. In this context the 'uncorrelated' phase might gain some physical meaning.

The situation is more difficult in the 'collapsed' phase ' $\mathrm{B}$ '. Inside this phase the transfer matrix can be parametrized as follows:

$$
\begin{aligned}
&\left\langle n\left|M_{B}\right| m\right\rangle=\mathcal{N}[n+m][\exp (\left.-\frac{\left((m-n)-\left[c_{0}\left(n+m-s^{b}\right)\right]_{+}\right)^{2}}{\Gamma\left(n+m-2 n_{0}\right)}\right) \\
&\left.+\exp \left(-\frac{\left((m-n)+\left[c_{0}\left(n+m-s^{b}\right)\right]_{+}\right)^{2}}{\Gamma\left(n+m-2 n_{0}\right)}\right)\right]
\end{aligned}
$$


where: $[.]_{+}=\max (., 0)$. The properties of the spatial volume distribution depend strongly on the parameter $c_{0}$, while a new kind of phase transition seems to be related with the $s^{b} \rightarrow \infty$ limit. We showed by direct measurement that this kind of phase transition occurs for the $\Delta$ coupling constant considerably larger than the $\Delta$ value where the 'traditional' 'B'-'C' phase transition found in previous studies occurs. It points to a new 'bifurcation' phase separating the ' $\mathrm{B}$ ' and ' $\mathrm{C}$ ' phases. That such a putative phase is not an artifact of the effective transfer matrix model is supported by measurements performed in the full CDT theory. They show that the scaling of the spatial volume distribution $n_{t}$ as a function of the total four-volume $\bar{N}_{41}$ changes when we get close to the 'old' 'B'-'C' transition. Deep in phase ' $\mathrm{C}$ ' the scaling is canonical, i.e. $n_{t} \propto \bar{N}_{41}^{3 / 4}$ for spatial slices in the 'blob', but in the new phase the scaling seems to be closer to $n_{t} \propto \bar{N}_{41}$. Such a different scaling of the spatial volume distribution and thus also of the time extent of the "blob" constituting the "physical" universe is interesting. It indicates that the 'B'- $C$ ' phase transition line might be associated with an asymmetric scaling of space and time, precisely as is assumed in Hořava-Lifshitz gravity [49, 50]. The new 'bifurcation' phase could be a genuinely new phase separating the ' $\mathrm{B}$ ' phase from the "ordinary" ' $\mathrm{C}$ ' phase. The exact nature of this new phase and its transition regions deserve further studies.

\section{Acknowledgments}

JG-S acknowledges the Polish National Science Centre (NCN) support via the grant DEC2012/05/N/ST2/02698. JA and AG were supported by the ERC-Advance grant 291092, "Exploring the Quantum Universe" (EQU). JA acknowledges support of FNU, the Free Danish Research Council, from the grant "quantum gravity and the role of black holes". JA was supported in part by Perimeter Institute of Theoretical Physics. Research at Perimeter Institute is supported by the Government of Canada through Industry Canada and by the Province of Ontario through the Ministry of Economic Development \& Innovation.

The authors thank Daniel Coumbe for careful proofreading and fruitful discussions.

Open Access. This article is distributed under the terms of the Creative Commons Attribution License (CC-BY 4.0), which permits any use, distribution and reproduction in any medium, provided the original author(s) and source are credited.

\section{References}

[1] T. Regge, General relativity without coordinates, Nuovo Cim. 19 (1961) 558 [INSPIRE].

[2] F. David, Planar diagrams, two-dimensional lattice gravity and surface models, Nucl. Phys. B 257 (1985) 45 [INSPIRE].

[3] V.A. Kazakov, A.A. Migdal and I.K. Kostov, Critical properties of randomly triangulated planar random surfaces, Phys. Lett. B 157 (1985) 295 [INSPIRE].

[4] J. Ambjørn, B. Durhuus and J. Fröhlich, Diseases of triangulated random surface models and possible cures, Nucl. Phys. B 257 (1985) 433 [InSPIRE].

[5] J. Ambjorn, B. Durhuus, J. Frohlich and P. Orland, The appearance of critical dimensions in regulated string theories, Nucl. Phys. B 270 (1986) 45. 
[6] D.V. Boulatov, V.A. Kazakov, I.K. Kostov and A.A. Migdal, Analytical and numerical study of the model of dynamically triangulated random surfaces, Nucl. Phys. B 275 (1986) 641 [INSPIRE].

[7] V.G. Knizhnik, A.M. Polyakov and A.B. Zamolodchikov, Fractal structure of $2 D$ quantum gravity, Mod. Phys. Lett. A 3 (1988) 819 [INSPIRE].

[8] F. David, Conformal field theories coupled to $2 D$ gravity in the conformal gauge, Mod. Phys. Lett. A 3 (1988) 1651 [INSPIRE].

[9] J. Distler and H. Kawai, Conformal field theory and 2D quantum gravity or who's afraid of Joseph Liouville?, Nucl. Phys. B 321 (1989) 509 [inSPIRE].

[10] J. Ambjørn and K.N. Anagnostopoulos, Quantum geometry of 2D gravity coupled to unitary matter, Nucl. Phys. B 497 (1997) 445 [hep-lat/9701006] [INSPIRE].

[11] J. Ambjørn and S. Varsted, Entropy estimate in three-dimensional simplicial quantum gravity, Phys. Lett. B 266 (1991) 285 [INSPIRE].

[12] M.E. Agishtein and A.A. Migdal, Three-dimensional quantum gravity as dynamical triangulation, Mod. Phys. Lett. A 6 (1991) 1863 [Erratum ibid. A 6 (1991) 2555] [InSPIRE].

[13] D.V. Boulatov and A. Krzywicki, On the phase diagram of three-dimensional simplicial quantum gravity, Mod. Phys. Lett. A 6 (1991) 3005 [inSPIRE].

[14] J. Ambjørn, D.V. Boulatov, A. Krzywicki and S. Varsted, The vacuum in three-dimensional simplicial quantum gravity, Phys. Lett. B 276 (1992) 432 [INSPIRE].

[15] J. Ambjørn and S. Varsted, Three-dimensional simplicial quantum gravity, Nucl. Phys. B 373 (1992) 557 [INSPIRE].

[16] J. Ambjørn and J. Jurkiewicz, Four-dimensional simplicial quantum gravity, Phys. Lett. B 278 (1992) 42 [INSPIRE].

[17] M.E. Agishtein and A.A. Migdal, Simulations of four-dimensional simplicial quantum gravity, Mod. Phys. Lett. A 7 (1992) 1039 [inSPIRE].

[18] S. Bilke et al., 4-D simplicial quantum gravity interacting with gauge matter fields, Phys. Lett. B 418 (1998) 266 [hep-lat/9710077] [INSPIRE].

[19] S. Bilke et al., 4-D simplicial quantum gravity: matter fields and the corresponding effective action, Phys. Lett. B 432 (1998) 279 [hep-lat/9804011] [INSPIRE].

[20] J. Ambjørn, K.N. Anagnostopoulos and J. Jurkiewicz, Abelian gauge fields coupled to simplicial quantum gravity, JHEP 08 (1999) 016 [hep-lat/9907027] [INSPIRE].

[21] S. Horata, H.S. Egawa, N. Tsuda and T. Yukawa, Phase structure of four-dimensional simplicial quantum gravity with a U(1) gauge field, Prog. Theor. Phys. 106 (2001) 1037 [hep-lat/0004021] [INSPIRE].

[22] P. Bialas, Z. Burda, A. Krzywicki and B. Petersson, Focusing on the fixed point of $4 D$ simplicial gravity, Nucl. Phys. B 472 (1996) 293 [hep-lat/9601024] [INSPIRE].

[23] B.V. de Bakker, Further evidence that the transition of $4 D$ dynamical triangulation is first order, Phys. Lett. B 389 (1996) 238 [hep-lat/9603024] [INSPIRE].

[24] S. Catterall, R. Renken and J.B. Kogut, Singular structure in 4D simplicial gravity, Phys. Lett. B 416 (1998) 274 [hep-lat/9709007] [INSPIRE]. 
[25] J. Ambjørn and R. Loll, Nonperturbative Lorentzian quantum gravity, causality and topology change, Nucl. Phys. B 536 (1998) 407 [hep-th/9805108] [INSPIRE].

[26] J. Ambjørn, J. Jurkiewicz and R. Loll, A nonperturbative lorentzian path integral for gravity, Phys. Rev. Lett. 85 (2000) 924 [hep-th/0002050] [inSPIRE].

[27] J. Ambjørn, J. Jurkiewicz and R. Loll, Nonperturbative 3D lorentzian quantum gravity, Phys. Rev. D 64 (2001) 044011 [hep-th/0011276] [INSPIRE].

[28] J. Ambjørn, J. Jurkiewicz and R. Loll, Dynamically triangulating lorentzian quantum gravity, Nucl. Phys. B 610 (2001) 347 [hep-th/0105267] [InSPIRE].

[29] J. Ambjørn, A. Görlich, J. Jurkiewicz and R. Loll, Nonperturbative quantum gravity, Phys. Rept. 519 (2012) 127 [arXiv: 1203.3591] [INSPIRE].

[30] J. Ambjørn, J. Jurkiewicz and R. Loll, Quantum gravity as sum over spacetimes, Lect. Notes Phys. 807 (2010) 59 [arXiv:0906.3947] [INSPIRE].

[31] J. Ambjørn, A. Görlich, J. Jurkiewicz and R. Loll, CDT - An entropic theory of quantum gravity, arXiv: 1007.2560 [INSPIRE].

[32] J. Ambjørn, J. Jurkiewicz and R. Loll, The Universe from scratch, Contemp. Phys. 47 (2006) 103 [hep-th/0509010] [INSPIRE].

[33] R. Loll, The emergence of spacetime or quantum gravity on your desktop, Class. Quant. Grav. 25 (2008) 114006 [arXiv:0711.0273] [INSPIRE].

[34] J. Ambjørn, J. Jurkiewicz and R. Loll, Reconstructing the universe, Phys. Rev. D 72 (2005) 064014 [hep-th/0505154] [INSPIRE].

[35] J. Ambjørn, A. Görlich, S. Jordan, J. Jurkiewicz and R. Loll, CDT meets Hoŕava-Lifshitz gravity, Phys. Lett. B 690 (2010) 413 [arXiv:1002.3298] [INSPIRE].

[36] J. Ambjørn, J. Jurkiewicz and R. Loll, Emergence of a $4 D$ world from causal quantum gravity, Phys. Rev. Lett. 93 (2004) 131301 [hep-th/0404156] [INSPIRE].

[37] J. Ambjørn, J. Jurkiewicz and R. Loll, Semiclassical universe from first principles, Phys. Lett. B 607 (2005) 205 [hep-th/0411152] [INSPIRE].

[38] J. Ambjørn, J. Jurkiewicz and R. Loll, Reconstructing the universe, Phys. Rev. D 72 (2005) 064014 [hep-th/0505154] [INSPIRE].

[39] J. Ambjørn, A. Görlich, J. Jurkiewicz and R. Loll, Planckian birth of the quantum de Sitter universe, Phys. Rev. Lett. 100 (2008) 091304 [arXiv: 0712.2485] [INSPIRE].

[40] J. Ambjørn, J. Jurkiewicz and R. Loll, The self-organized de Sitter universe, Int. J. Mod. Phys. D 17 (2009) 2515 [arXiv: 0806. 0397] [INSPIRE].

[41] J. Ambjørn, A. Görlich, J. Jurkiewicz and R. Loll, Geometry of the quantum universe, Phys. Lett. B 690 (2010) 420 [arXiv: 1001.4581] [INSPIRE].

[42] J. Ambjørn, A. Görlich, J. Jurkiewicz and R. Loll, The nonperturbative quantum de Sitter universe, Phys. Rev. D 78 (2008) 063544 [arXiv:0807.4481] [INSPIRE].

[43] J. Ambjørn, et al., The semiclassical limit of causal dynamical triangulations, Nucl. Phys. B 849 (2011) 144 [arXiv:1102.3929] [INSPIRE].

[44] J. Ambjørn, J. Gizbert-Studnicki, A. Görlich and J. Jurkiewicz, The transfer matrix in four-dimensional CDT, JHEP 09 (2012) 017 [arXiv: 1205.3791] [INSPIRE]. 
[45] J. Ambjørn, S. Jordan, J. Jurkiewicz and R. Loll, A second-order phase transition in CDT, Phys. Rev. Lett. 107 (2011) 211303 [arXiv:1108.3932] [INSPIRE].

[46] J. Ambjørn, S. Jordan, J. Jurkiewicz and R. Loll, Second- and first-order phase transitions in CDT, Phys. Rev. D 85 (2012) 124044 [arXiv:1205.1229] [INSPIRE].

[47] L. Bogacz, Z. Burda and B. Waclaw, Quantum widening of CDT universe, Phys. Rev. D 86 (2012) 104015 [arXiv:1204.1356] [INSPIRE].

[48] J. Mielczarek, Asymptotic silence in loop quantum cosmology, AIP Conf. Proc. 1514 (2012) 81 [arXiv: 1212.3527] [INSPIRE].

[49] P. Hořava, Quantum gravity at a Lifshitz point, Phys. Rev. D 79 (2009) 084008 [arXiv:0901.3775] [INSPIRE].

[50] P. Hořava and C.M. Melby-Thompson, General covariance in quantum gravity at a Lifshitz point, Phys. Rev. D 82 (2010) 064027 [arXiv: 1007.2410] [InSPIRE]. 